ESAIM: M2AN 49 (2015) 19-37

DOI: $10.1051 / \mathrm{m} 2 \mathrm{an} / 2014023$
ESAIM: Mathematical Modelling and Numerical Analysis

www.esaim-m2an.org

\title{
ON THE NUMERICAL INTEGRATION OF SCALAR NONLOCAL CONSERVATION LAWS
}

\author{
Paulo Amorim ${ }^{1}$, Rinaldo M. Colombo ${ }^{2}$ And Andreia Teixeira ${ }^{3}$
}

\begin{abstract}
We study a rather general class of 1D nonlocal conservation laws from a numerical point of view. First, following [F. Betancourt, R. Bürger, K.H. Karlsen and E.M. Tory, On nonlocal conservation laws modelling sedimentation. Nonlinearity 24 (2011) 855-885], we define an algorithm to numerically integrate them and prove its convergence. Then, we use this algorithm to investigate various analytical properties, obtaining evidence that usual properties of standard conservation laws fail in the nonlocal setting. Moreover, on the basis of our numerical integrations, we are led to conjecture the convergence of the nonlocal equation to the local ones, although no analytical results are, to our knowledge, available in this context.
\end{abstract}

Mathematics Subject Classification. 35L65.

Received July 2, 2013. Revised April 16, 2014.

Published online January 12, 2015.

\section{INTRODUCTION}

Conservation laws with nonlocal fluxes have appeared recently in the literature, arising naturally in many fields of application, such as in crowd dynamics (see $[6-8,22]$ and the references therein), in models inspired from biology, see $[5,12,13,15]$ and, more recently, also in an industrial context [16].

In this paper, we aim at the study of these equations from a numerical point of view. First, we prove the convergence of a finite volume algorithm to numerically integrate a class of one-dimensional conservation laws with a nonlocal flow. Then, we use this algorithm to show peculiar properties of these nonlocal equations and, in particular, how they differ from the usual local ones.

Consider the scalar equation

$$
\left\{\begin{array}{l}
\partial_{t} \rho+\partial_{x}(f(t, x, \rho) v(\rho * \eta))=0 \\
\rho(0, x)=\rho^{o}(x)
\end{array} \quad(t, x) \in \mathbb{R}^{+} \times \mathbb{R}\right.
$$

which slightly extends, in the 1D case, the class of equations considered in [2,7,8]. Slightly extending [2], the numerical scheme below allows to integrate (1.1) and we prove its convergence. As a byproduct, we also establish an existence result for (1.1), thus slightly extending also ([7], Thm. 2.2) in the 1D case.

Keywords and phrases. Nonlocal conservation laws, Lax Friedrichs scheme.

1 Instituto de Matemática, Universidade Federal do Rio de Janeiro, C.P. 68530, Cidade Universitária 21945-970, Rio de Janeiro, Brazil.

2 Unità INdAM, Università di Brescia, Via Branze 38, 25123 Brescia, Italy. rinaldo@ing.unibs.it

3 Centro de Matemática e Aplicações Fundamentais, Departamento de Matemática, Universidade de Lisboa, Av. Prof. Gama Pinto 2, 1649-003 Lisboa, Portugal. 
In the case $f(t, x, \rho)=\rho(1-\rho)^{\alpha},(1.1)$ reduces to the case considered in [2], motivated by a sedimentation model, see also [1].

This numerical algorithm is implemented and then used to investigate various properties of (1.1). First, we provide evidence that the usual Maximum Principle for scalar conservation laws fails in the case of (1.1), see $[4,14,21]$ in the different context of Vlasov-Poisson-Fokker-Planck systems. Another integration shows that the total variation of the solution to (1.1) may well sharply increase, contrary to what happens in the standard local situation. Remark that both these examples are in agreement with the estimates we rigorously obtain on the approximate solutions.

Of particular interest is the limit $\eta \rightarrow \delta, \delta$ being the Dirac measure centered at the origin. Numerical integrations apparently show that the solutions to (1.1) converge to its formal limit, namely

$$
\left\{\begin{array}{l}
\partial_{t} \rho+\partial_{x}(f(t, x, \rho) v(\rho))=0 \\
\rho(0, x)=\rho^{o}(x)
\end{array} \quad(t, x) \in \mathbb{R}^{+} \times \mathbb{R}\right.
$$

although no rigorous proof of this convergence is, to our knowledge, known. Remark that in the nonlocal case, well posedness results are available also in the case of systems in several space dimensions, see $[8,10]$. Hence, the ability of passing to the limit $\eta \rightarrow \delta$ might help in the search for analytical results about systems of conservation laws in several space dimensions.

The scheme below has an associated CFL condition. The CFL condition is often interpreted through a comparison between the numerical propagation speed and the analytical one, see for instance [19], Section 4.4, page 68 . In the present nonlocal case (1.1), information propagates at an infinite speed, due to the presence of the term $\eta * \rho$. Nevertheless, also in the nonlocal case (1.1) a suitable CFL condition plays a key role, see (2.3).

Second, the scheme below is not monotone in the sense of the usual definition ([19], formula (12.42)), as follows from the integration in Section 3.2. There, both constant initial data $\bar{\rho}=0$ and $\bar{\rho}=1$ yield constant solutions, but the initial datum (3.5), although it attains values in $[0,1]$, yields a solution exceeding 1 . Nevertheless, the scheme (2.5) enjoys several properties of monotone schemes, proved in the lemmas in Section 2.

Remark 1.1. Throughout this work, we follow the usual habit of referring to (1.1) as to a nonlocal equation and, hence, to the standard case (1.2) as to the local case. However, whenever the support of $\eta$ is bounded, it might seem more appropriate to call (1.1) a local equation and (1.2) the punctual case.

The next section deals with the definition of the algorithm and with the statement of the estimates which ensure its convergence, as well as the entropicity of the limit solution. Section 3 deals with various numerical integrations of (1.1). All proofs are deferred to the last Section 4.

\section{MAIN RESULTS}

Throughout, we set $\mathbb{R}^{+}=[0,+\infty[$.

As a starting point, we state what we mean by solution to (1.1), see also ([7], Def. 2.1).

Definition 2.1. Let $T>0$. Fix $\rho^{o} \in \mathbf{L}^{\infty}(\mathbb{R} ; \mathbb{R})$. A weak entropy solution to $(1.1)$ on $[0, T]$ is a bounded measurable Kružkov solution $\rho \in \mathbf{C}^{0}\left([0, T] ; \mathbf{L}_{l o c}^{1}(\mathbb{R} ; \mathbb{R})\right)$ to

$$
\left\{\begin{array}{l}
\partial_{t} \rho+\partial_{x}(f(t, x, \rho) V(t, x))=0 \\
\rho(0, x)=\rho^{o}(x)
\end{array} \quad \text { where } \quad V(t, x)=v((\rho(t) * \eta)(x)) .\right.
$$

For the definition of Kružkov solution, see for instance ([11], Sect. 6.2) or ([18], Def. 1). Here, as usual,

$$
(\rho(t) * \eta)(x)=\int_{\mathbb{R}} \rho(t, \xi) \eta(x-\xi) \mathrm{d} \xi .
$$


Remark that the assumptions

$$
\begin{gathered}
f \in \mathbf{C}^{2}\left(\mathbb{R}^{+} \times \mathbb{R} \times \mathbb{R} ; \mathbb{R}\right) \text { and }\left\{\begin{array}{c}
\sup _{t, x, \rho}\left|\partial_{\rho} f(t, x, \rho)\right|<+\infty \\
\sup _{t, x}\left|\partial_{x} f(t, x, \rho)\right|<C|\rho| \\
\sup _{t, x}\left|\partial_{x x}^{2} f(t, x, \rho)\right|<C|\rho| \\
\forall t, x \quad f(t, x, 0)=0
\end{array}\right. \\
v \in\left(\mathbf{C}^{2} \cap \mathbf{W}^{1, \infty}\right)(\mathbb{R} ; \mathbb{R}) \text { and } \quad \eta \in\left(\mathbf{C}^{2} \cap \mathbf{W}^{2, \infty}\right)(\mathbb{R} ; \mathbb{R})
\end{gathered}
$$

ensure that the transport equation in Definition 2.1 fits in Kružkov framework, see [11,18]. From the modeling point of view, it is natural to require that the kernel $\eta$ attains only positive (or non-negative) values. However, this requirement is not necessary for the analytical results below.

Below, Remark 2.3 and Lemma 2.5 provide uniform $\mathbf{L}^{\infty}$ bounds on the solution to (1.1) under conditions (2.1)-(2.2) on the equations and for data in $\mathbf{L}^{\infty}$. Therefore, the apparently strong requirement $\left\|\partial_{\rho} f\right\|_{\mathbf{L}^{\infty}}<+\infty$ can be easily relaxed to

$$
\sup _{t \in \mathbb{R}^{+}, x \in \mathbb{R}, \rho \in[-M, M]}\left|\partial_{\rho} f(t, x, \rho)\right|<+\infty
$$

for a suitable positive $M$. Moreover, the usual sublinearity condition $\sup _{t, x}\left|\partial_{x} f(t, x, \rho)\right|<C(1+|\rho|)$ takes the form $\sup _{t, x}\left|\partial_{x} f(t, x, \rho)\right|<C|\rho|$ in (2.1) due to the assumption $f(t, x, 0)=0$ for all $t$ and $x$.

Introduce a uniform mesh with size $h$ along the $x$ axis and size $\tau$ along the $t$ axis. Throughout, we assume that the following $C F L$ condition is satisfied:

$$
\lambda\left(C h+2\left\|\partial_{\rho} f\right\|_{\mathbf{L}^{\infty}}\right)\|v\|_{\mathbf{L}^{\infty}} \leq \frac{1}{3}
$$

with $C$ as in (2.1) and where, as usual, $\lambda=\tau / h$. Although this CFL condition is somewhat more restrictive than in the local case, it has the advantage that in the proofs of the main stability estimates below, one can maintain the complexity of the proofs to a minimum. Moreover, (2.3) is not optimal in the sense that it does not formally reduce to the usual CFL condition for the Lax-Friedrichs scheme in a local setting. However, our strategy was to consider a simple numerical method for which calculations can be carried out with the utmost clarity (leading us to choose the Lax-Friedrichs method), but for which the novel, nonlocal effects could also be accounted for. Thus it would be interesting to optimize the calculations in the proofs below in order to reach a more efficient CFL condition, although this falls outside the scope of this paper.

Consider the following Lax-Friedrichs type scheme:

$$
\left\{\begin{array}{l}
\rho_{j}^{n+1}=\rho_{j}^{n}-\lambda\left(\mathbf{f}_{j+1 / 2}^{n}\left(\rho_{j}^{n}, \rho_{j+1}^{n}\right)-\mathbf{f}_{j-1 / 2}^{n}\left(\rho_{j-1}^{n}, \rho_{j}^{n}\right)\right) \\
\rho_{j}^{o}=\frac{1}{h} \int_{x_{j-1 / 2}}^{x_{j+1 / 2}} \rho^{o}(x) \mathrm{d} x
\end{array}\right.
$$

where the numerical flux $\mathbf{f}_{j+1 / 2}^{n}$ in $(2.4)$ is given by

$$
\mathbf{f}_{j+1 / 2}^{n}\left(\rho_{1}, \rho_{2}\right):=\frac{f\left(t^{n}, x_{j+1 / 2}, \rho_{1}\right)+f\left(t^{n}, x_{j+1 / 2}, \rho_{2}\right)}{2} v\left(c_{j+1 / 2}^{n}\right)-\frac{1}{6 \lambda}\left(\rho_{2}-\rho_{1}\right) .
$$

Here, the convolution is computed through a standard quadrature formula using the same space mesh, as follows

$$
c_{j+1 / 2}^{n}=\sum_{k \in \mathbb{Z}} h \rho_{k+1 / 2}^{n} \eta_{j+1 / 2-k}
$$

where $\rho_{k+1 / 2}^{n}$ is any convex combination of $\rho_{k}^{n}, \rho_{k+1}^{n}$ and, for instance, $\eta_{j+1 / 2}=\frac{1}{h} \int_{x_{j}}^{x_{j+1}} \eta(x) \mathrm{d} x$, for instance. 
The next three lemmas provide the basic properties of the algorithm (2.4), namely positivity, $\mathbf{L}^{1}$ and $\mathbf{L}^{\infty}$ bounds. All proofs are deferred to Section 4.

Lemma 2.2 (Positivity). Let conditions (2.1)-(2.2) hold. Assume that $h$ and $\tau$ satisfy the CFL condition (2.3). If $\rho_{j}^{o} \geq 0$ for all $j$, then the approximate solution constructed by the algorithm (2.4) is such that $\rho_{j}^{n} \geq 0$ for all $j$ and $n$.

Remark 2.3. The proof of the above lemma clearly shows that if we assume $\rho^{o} \leq 0$, then $\rho^{n} \leq 0$ for all $n$. Moreover, under the same assumptions (2.1)-(2.2)-(2.3), a straightforward modification of the proof of Lemma 2.2 ensures that if there exists a $\bar{\rho} \in \mathbb{R}^{+}$such that $f(t, x, \bar{\rho})=0$, then the inequality $\rho^{o} \geq \bar{\rho}$, respectively $\rho^{o} \leq \bar{\rho}$, implies that $\rho^{n} \geq \bar{\rho}$, respectively $\rho^{n} \leq \bar{\rho}$, for all $n$.

Lemma 2.4 ( $\mathbf{L}^{1}$ bound). Let conditions (2.1)-(2.2) hold. Assume that $h$ and $\tau$ satisfy the CFL condition (2.3). If $\rho_{j}^{o} \geq 0$ for all $j$, then the approximate solution constructed by the algorithm (2.4) satisfies

$$
\left\|\rho^{n}\right\|_{\mathbf{L}^{1}} \leq\left\|\rho^{o}\right\|_{\mathbf{L}^{1}} .
$$

Lemma 2.5 ( $\mathbf{L}^{\infty}$ bound). Let conditions (2.1)-(2.2) hold. Assume that $h$ and $\tau$ satisfy the CFL condition (2.3). If $\rho_{j}^{o} \geq 0$ for all $j$, then the solution constructed by the algorithm (2.4) satisfies

$$
\left\|\rho^{n}\right\|_{\mathbf{L}^{\infty}} \leq\left\|\rho^{o}\right\|_{\mathbf{L}^{\infty}} \mathrm{e}^{\mathcal{L} t}
$$

where $\mathcal{L}$ depends on $C$ in (2.1), on various norms of $f, v, \eta$ and on the $\mathbf{L}^{1}$ norm of the initial datum, see (4.4).

The next result concerns the bound on the total variation of the approximate solution constructed in (2.4). In the standard Kružkov case, when the flow is independent from $t$ and $x$, the total variation of the solution is well know to be a non-increasing function of time, see ([3], Thm. 6.1). Here, on the contrary, the total variation and the $\mathbf{L}^{\infty}$ norm of the solution to (1.1) may well sharply increase due to the nonlocal terms, even when the flow is independent from $t$ and $x$, see Section 3.2.

Proposition 2.6 (Total variation bound). Let conditions (2.1)-(2.2) hold. Assume that $h$ and $\tau$ satisfy the CFL condition (2.3). If $\rho_{j}^{o} \geq 0$ for all $j$, then the approximate solution constructed by the algorithm (2.4) satisfies the following total variation estimate, for all $n \geq 0$ :

$$
\sum_{j \in \mathbb{Z}}\left|\rho_{j+1}^{n}-\rho_{j}^{n}\right| \leq \mathrm{e}^{\mathcal{K}_{1} t} \sum_{j \in \mathbb{Z}}\left|\rho_{j+1}^{o}-\rho_{j}^{o}\right|+\frac{\mathrm{e}^{\mathcal{K}_{1} t}-1}{\mathcal{K}_{1}} \mathcal{K}_{2},
$$

where the constants $\mathcal{K}_{1}$ and $\mathcal{K}_{2}$ depend on $C$ in (2.1), on various norms of $f, v, \eta$ and of the initial datum, see (4.14).

As the explicit expressions in (4.14) show, in the standard case of the local homogeneous conservation law $\partial_{t} \rho+\partial_{x} f(\rho)=0$, we have $v \equiv 1, \eta=0, C=0$ and (2.7) reduces to the usual total variation diminishing condition $\sum_{j \in \mathbb{Z}}\left|\rho_{j+1}^{n}-\rho_{j}^{n}\right| \leq \sum_{j \in \mathbb{Z}}\left|\rho_{j+1}^{o}-\rho_{j}^{o}\right|$. We recall also that the form of (2.7) is consistent with that in Theorem 2.5 of [9].

A first consequence of the bound on the total variation is the $\mathbf{L}^{1}$-Lipschitz continuity in time of the approximate solution, proved in the following lemma.

Lemma 2.7 ( $\mathbf{L}^{1}$-Lipschitz continuity in time). Fix a positive T. Let conditions (2.1)-(2.2) hold. Fix strictly positive $h$ and $\tau$ satisfying the CFL condition (2.3). If $\rho_{j}^{o} \geq 0$ for all $j$, then the approximate solution constructed by the algorithm (2.4) is an $\mathbf{L}^{1}$-Lipschitz continuous function of time, in the sense that for any $n, m \in \mathbb{N}$ such that $n \tau \leq T$ and $m \tau \leq T$,

$$
\left\|\rho^{n}-\rho^{m}\right\|_{\mathbf{L}^{1}} \leq \mathcal{C}(T)|n-m| \tau
$$

where the quantity $\mathcal{C}(T)$ grows exponentially in time and depends on $C$ in $(2.1)$, on various norms of $f, v, \eta$ and of the initial datum, see (4.15). 
The $\mathbf{L}^{\infty}$ bound proved in Lemma 2.5, the total variation bound proved in Proposition 2.6 and the uniform continuity in time that follows from Lemma 2.7 allow to apply Helly Theorem, for instance in the form of Theorem 2.6 of [3], to the sequence of approximate solutions constructed through (2.4). A straightforward limiting procedure, see for instance ([3], Sect. 6.2), thus ensures the existence of weak solutions to the Cauchy problem for (1.1).

To obtain uniqueness, we prove that the approximate solutions (2.4) also satisfy a discrete entropy condition. To this end, define for each $k \in \mathbb{R}$ the Kružkov numerical entropy flux as

$$
F_{j+1 / 2}^{k}\left(\rho_{1}, \rho_{2}\right)=\mathbf{f}_{j+1 / 2}^{n}\left(\rho_{1} \vee k, \rho_{2} \vee k\right)-\mathbf{f}_{j+1 / 2}^{n}\left(\rho_{1} \wedge k, \rho_{2} \wedge k\right),
$$

where $a \vee b=\max (a, b)$ and $a \wedge b=\min (a, b)$.

Proposition 2.8 (Discrete entropy condition). Let conditions (2.1)-(2.2) hold. Assume that $h$ and $\tau$ satisfy the CFL condition (2.3). If $\rho_{j}^{o} \geq 0$ for all $j$, then the approximate solution constructed by the algorithm (2.4) verifies the discrete entropy inequality

$$
\begin{gathered}
\left|\rho_{j}^{n+1}-k\right|-\left|\rho_{j}^{n}-k\right|+\lambda\left(F_{j+1 / 2}^{k}\left(\rho_{j}^{n}, \rho_{j+1}^{n}\right)-F_{j-1 / 2}^{k}\left(\rho_{j-1}^{n}, \rho_{j}^{n}\right)\right) \\
+\lambda \operatorname{sgn}\left(\rho_{j}^{n+1}-k\right)\left(f\left(t^{n}, x_{j+1 / 2}, k\right) v\left(c_{j+1 / 2}\right)-f\left(t^{n}, x_{j-1 / 2}, k\right) v\left(c_{j-1 / 2}\right)\right) \leq 0
\end{gathered}
$$

for all $k \in \mathbb{R}$.

\section{NumERICAL INTEGRATIONS}

\subsection{A nonlocal traffic model}

The classical Lighthill-Whitham [20] and Richards [23] (LWR) model for vehicular traffic consists of the continuity equation $\partial_{t} \rho+\partial_{x}(\rho V)=0$ supplied with a suitable speed law $V=V(\rho)$. Here, as usual, $t$ is time, $x$ an abscissa along a rectilinear road with neither entries nor exits and $\rho \in[0,1]$ is the (average) vehicular density.

Equation (1.1) with

$$
f(\rho)=\rho(1-\rho), \quad v(r)=V_{\max }(1-r) \quad \text { and } \quad \eta(x)=\alpha((x-a)(b-x))^{5 / 2} \chi_{[a, b]}(x),
$$

where $V_{\max }>0$, can be used as an LWR-type macroscopic model for vehicular traffic, where drivers adjust their speed according to the local traffic density, so that the speed law takes the functional form

$$
V(\rho)=V_{\max }(1-\rho)(1-\rho * \eta) .
$$

The coefficient $\alpha$ in (3.1) is chosen so that $\int_{\mathbb{R}} \eta=1$. The parameters $a$ and $b$ are the horizon of each driver, in the sense that a driver situated at $x$ adjusts his speed according to the average vehicular density he sees on the interval $[x-b, x-a]$. To emphasize their roles, we select below the two situations

$$
\begin{array}{ll}
a=-1 / 4 \\
b=0
\end{array} \quad \text { and } \quad \begin{array}{ll}
a & =0 \\
b & =1 / 4 .
\end{array}
$$

In the former case, drivers look forward, while in the latter they look backward. We consider the initial datum

$$
\rho^{o}(x)=\frac{1}{2} \chi_{[-2.8,-1.8]}(x)+\frac{3}{4} \chi_{[-1.2,-0.2]}(x)+\frac{3}{4} \chi_{[0.6,1.0]}(x)+\chi_{[1.5,+\infty[}(x)
$$

representing three groups of vehicles lining up in a queue.

The results in Section 2 ensure that for any $\rho^{o} \in \mathbf{L}^{1}(\mathbb{R} ;[0,1])$, the Cauchy problem consisting of (1.1)-(3.1) with initial datum $\rho^{o}$ admits a unique solution $\rho=\rho(t, x)$ attaining values in $[0,1]$. However, the qualitative 

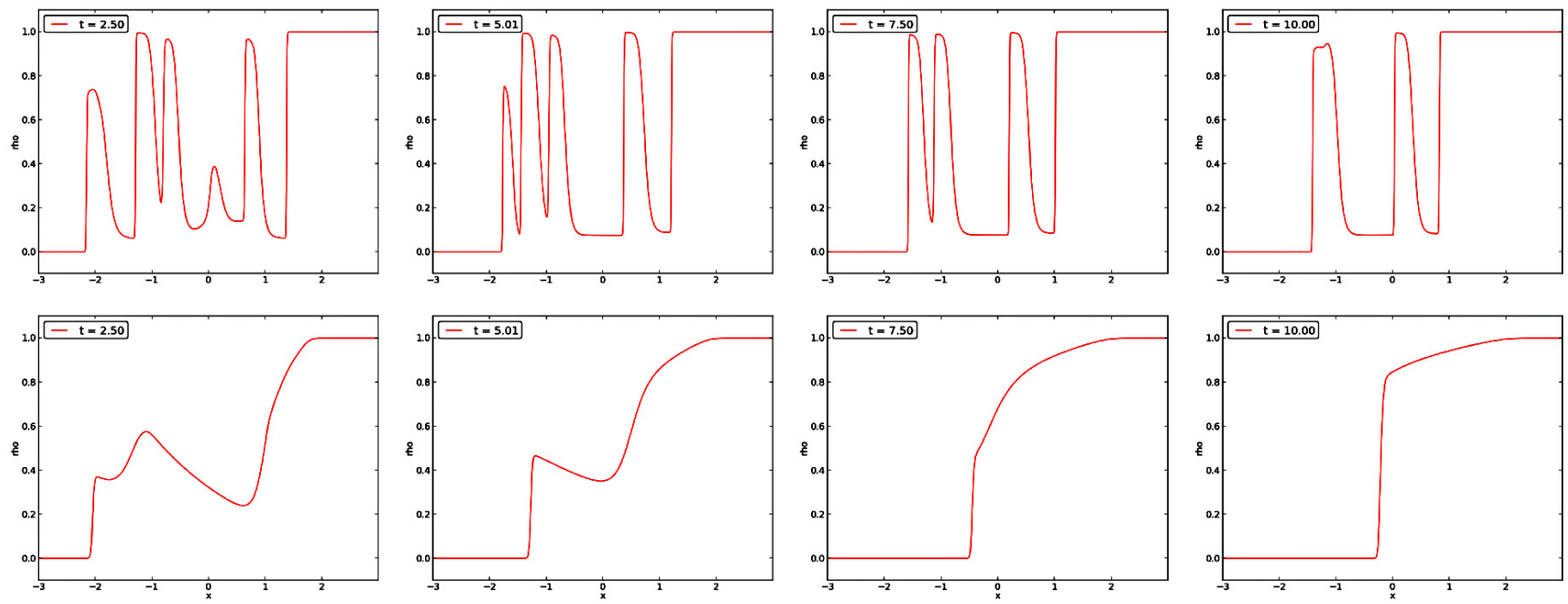

FiguRE 1. Integration of (1.1)-(3.1)-(3.3) in the two cases $(3.2)$ at times $t=2.50,5.01,7.50$, 10.00. Above, drivers look backward while below they look forward. Note, already on the first column, the difference in the two evolutions, clearly due to the position of the support of $\eta$. Here, the space mesh size is 0.0005 .

behaviors of the solutions are rather different in the two situations in (3.2), see Figure 1. Clearly, the evolution in the case of drivers looking forward (second line in Fig. 1) is far more reasonable, while the backward looking case leads to big oscillations in the vehicular density.

We observe that the particular choice (3.1), in view of Definition 2.1 and thanks to the Maximum Principle ([18], Thm. 3), ensures that an initial data $\rho^{o}$ attaining values in $[0,1]$ yields a solution also attaining values in the same interval.

\subsection{Increase of the total variation and of the $\mathrm{L}^{\infty}$ norm}

This paragraph is devoted to show that Lemma 2.2 and the total variation bound (2.7) are, at least qualitatively, optimal. Moreover, the example below shows that the nonlocal equation (1.1) does not enjoy two standard properties typical of 1D scalar conservation laws, namely the maximum principle ([3], (iv) in Thm. 6.3), see also ([18], Thm. 3), and the diminishing of the total variation ([3], Thm. 6.1).

In Remark 2.3 the assumption that $f(\bar{\rho})=0$ can not be replaced by $v(\bar{\rho})=0$ to ensure that the solution remains bounded between 0 and $\bar{\rho}$. Let $\bar{\rho}=1$ and consider (1.1) in the case

$$
f(\rho)=\rho, \quad v(r)=1-r \quad \text { and } \quad \eta(x)=\alpha((x-a)(b-x))^{5 / 2} \chi_{[a, b]}(x)
$$

with $\alpha$ chosen so that $\int_{\mathbb{R}} \eta=1$. Then, clearly, the initial data $\bar{\rho}(x) \equiv 1$ and $\bar{\rho}(x) \equiv 0$ are stationary solutions to (1.1)-(3.4). However, as the numerical integration below shows, the initial datum

$$
\rho^{o}(x)=0.25 \chi_{[-1.35,-0,95]}(x)+\chi_{[-0.85,-0.25]}(x)+0.75 \chi_{[-0.15, .25]}(x)
$$

which satisfies $\rho^{o}(x) \in[0,1]$ for all $x \in \mathbb{R}$, yields a solution $\rho=\rho(t, x)$ that exceeds $\bar{\rho}=1$, showing that (1.1)-(3.4) does not satisfy the Maximum Principle, see Figure 2.

Remark that the choice (3.4) leads to a flow in (1.1) which is independent both from $t$ and $x$. In the standard case of local scalar conservation laws, ([3], Thm. 6.1) ensures that the total variation of the solution may not 

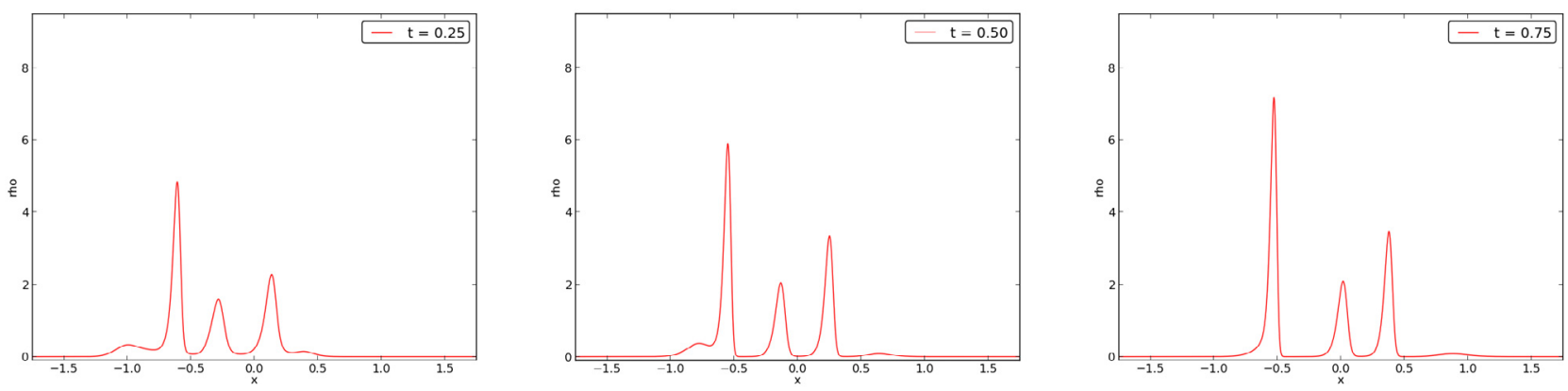

Figure 2. Numerical integration of (1.1)-(3.4) with $a=0, b=0.2$ and with the initial datum (3.5) at times $t=0.25,0.50,0.75$. The graph at time $t=1$ is in Figure 3, second row. Note the sharp increase in both the $\mathbf{L}^{\infty}$-norm and in the total variation. Here, the space mesh size is 0.0005 .
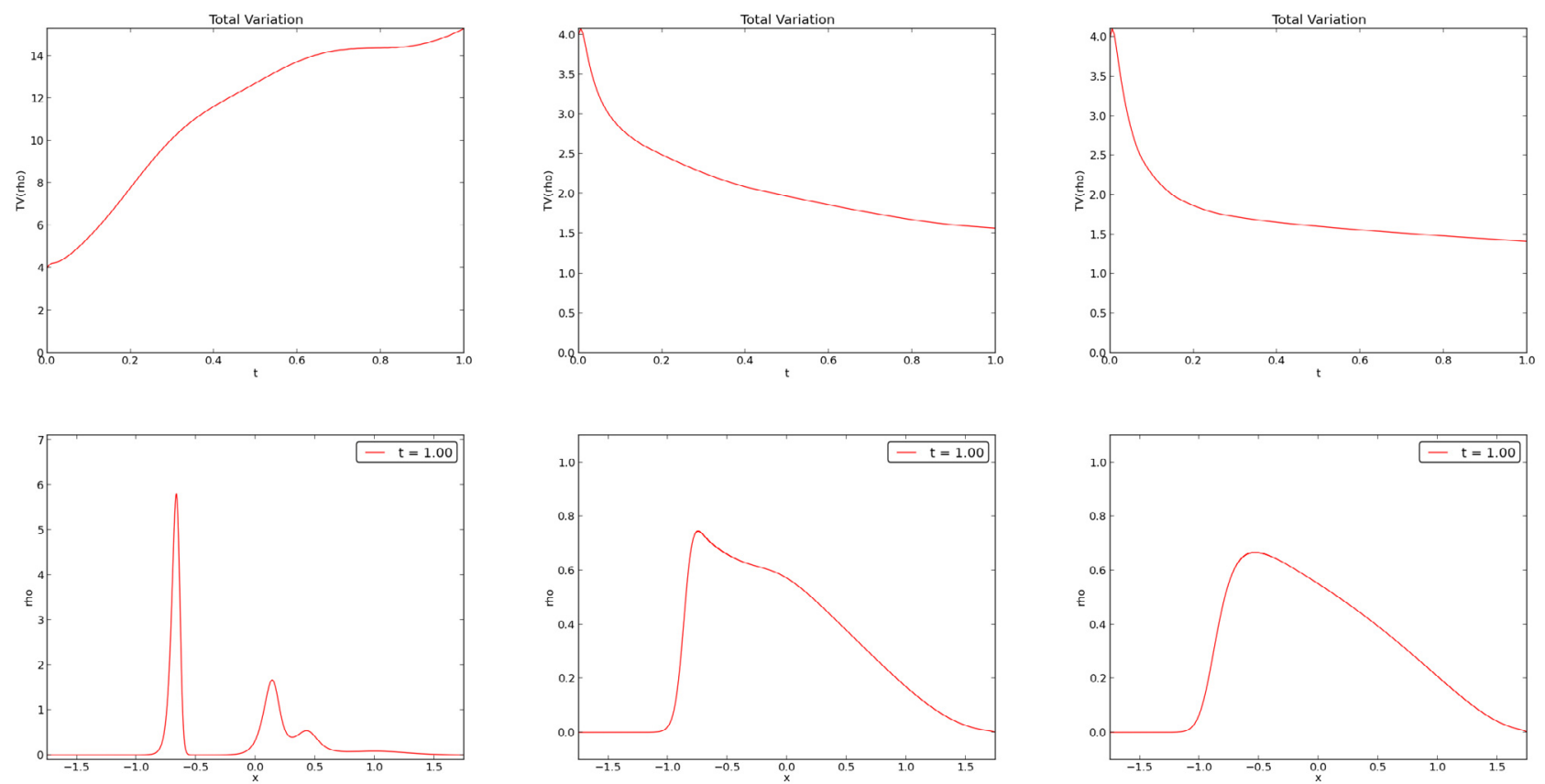

Figure 3. Above, total variation of the solution to (1.1)-(3.4)-(3.5) versus time, in the three cases $a=0, b=0.2 ; a=-0.1, b=0.1$ and $a=-0.2, b=0$. Below, graph of the solutions at time $t=1$. Remark that the vertical scales in the leftmost diagram differs from that used in the middle and on the right. Indeed, the initial total variation is the same, 4 , in all cases.

increase in time. The numerical integration below shows that the total variation of the solution to (1.1)-(3.4) may well sharply increase in a very short time, coherently with (2.7).

It is of interest to note that this behavior depends on the geometry of the support of $\eta$. Indeed, a translation of the convolution kernel leads to very different solutions, see Figure 3. When the support is contained in $\mathbb{R}^{+}$, there is a sharp increase in the total variation. In the other two cases, when spt $\eta$ is centered about the origin or contained in $\mathbb{R}^{-}$, there is a small increase in $\operatorname{TV}(\rho)$ for a small time interval, with a subsequent decrease. 

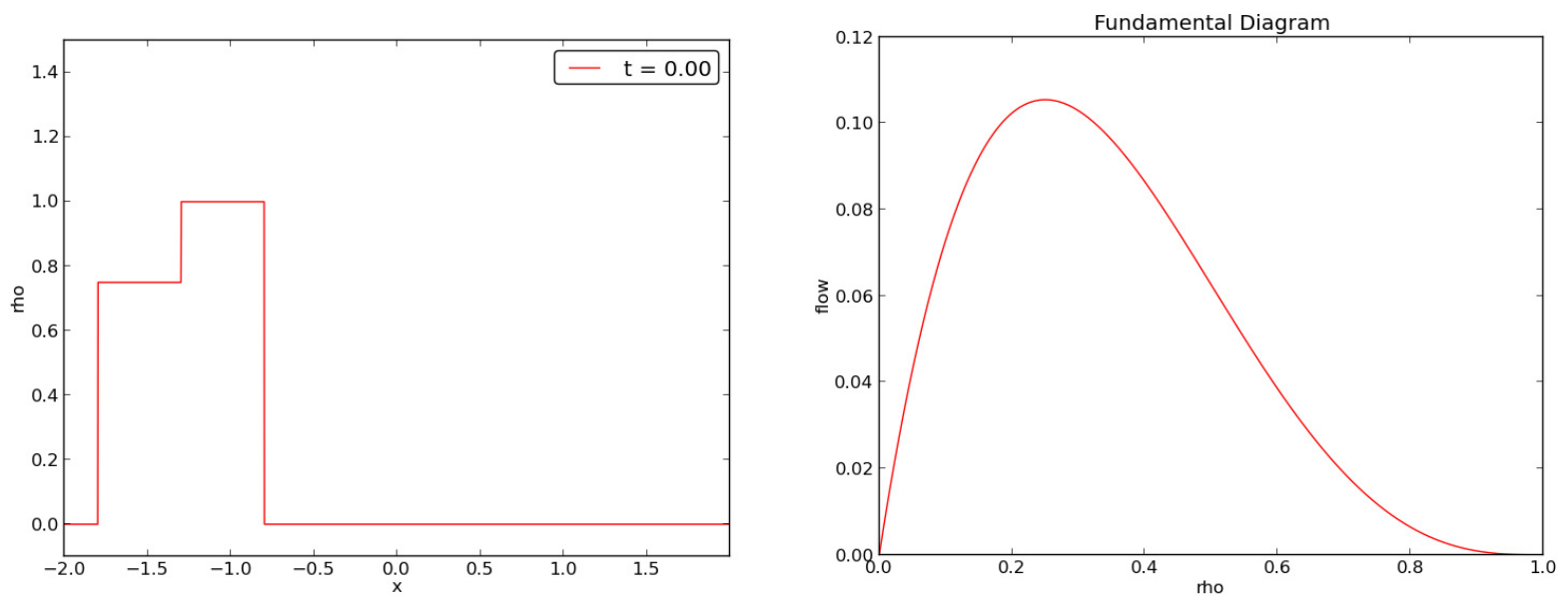

Figure 4. Left, the initial datum in (3.7) and, right, the flow (3.6) used in (1.2). Note its change of convexity.

\subsection{The nonlocal to local limit}

In this section we use the algorithm (2.4) to investigate the limit in which $\eta$ tends to a Dirac $\delta$, so that the nonlocal equation (1.1) tends, at least formally, to the local conservation law (1.2). To our knowledge, no analytical result is at present available on this limit.

Consider (1.1) with flow and speed

$$
f(t, x, \rho)=\rho, \quad v(r)=(1-r)^{3} \chi_{]-\infty, 1[}(r),
$$

see also Figure 4 left, while the convolution kernel and the initial datum are:

$$
\eta_{a}(x)=\alpha_{a}\left(a^{2}-x^{2}\right)^{5 / 2} \chi_{[-a, a]}(x), \quad \quad \rho_{o}(x)=\frac{3}{4} \chi_{[-1.8,-1.3[}(x)+\chi_{[-1.3,-0.8]}(x),
$$

and with the choices for $a=0.25, a=0.1, a=0.05$, with $\alpha_{a}$ computed so that $\int_{\mathbb{R}} \eta_{a}(x) \mathrm{d} x=1$. As limit case, we consider the standard conservation law (1.2) with $f$ and $v$ as in (3.6), see also Figure 4, right. In the integration below, the solution $\rho$ attains positive values, so that after an easy modification of $v$ on $\mathbb{R}^{-}$we can assume that (2.2) holds.

The resulting numerical integrations, carried out satisfying the CFL condition (2.3), give the diagrams in Figure 5. In the limit case of (1.2), the chosen initial datum leads to the formation of a rarefaction wave, a shock and a mixed wave, due to the change of convexity of the flow, see the lowest line in Figure 5 . To allow the comparison among these integrations, in all of them the same uniform mesh size was used, namely $\Delta x=0.0004$. This makes the computation of the integral in the nonlocal convolution term relatively less accurate at low values of $a$, nevertheless $\Delta x$ is sufficiently small to ensure that a sufficient number of mesh points enter the computation of the integral, also for small $a$. The numerical integrations shown in Figure 5 qualitatively suggest that in the limit $a \rightarrow 0$ the solution to (1.1)-(3.6)-(3.7) converges to that of (1.2)-(3.6). A more quantitative hint in this direction is in Figure 6. Using the algorithm above, we computed the solution $\rho_{a}$ to (1.1)-(3.6)-(3.7) for different values of $a$ and the solution $\rho$ to (1.2)-(3.6), all at time $t=0.500$. Figure 6 presents the plot of the $\mathbf{L}^{1}$-distance $\left\|\rho_{a}-\rho\right\|_{\mathbf{L}^{1}}$ versus $1 / a$. 

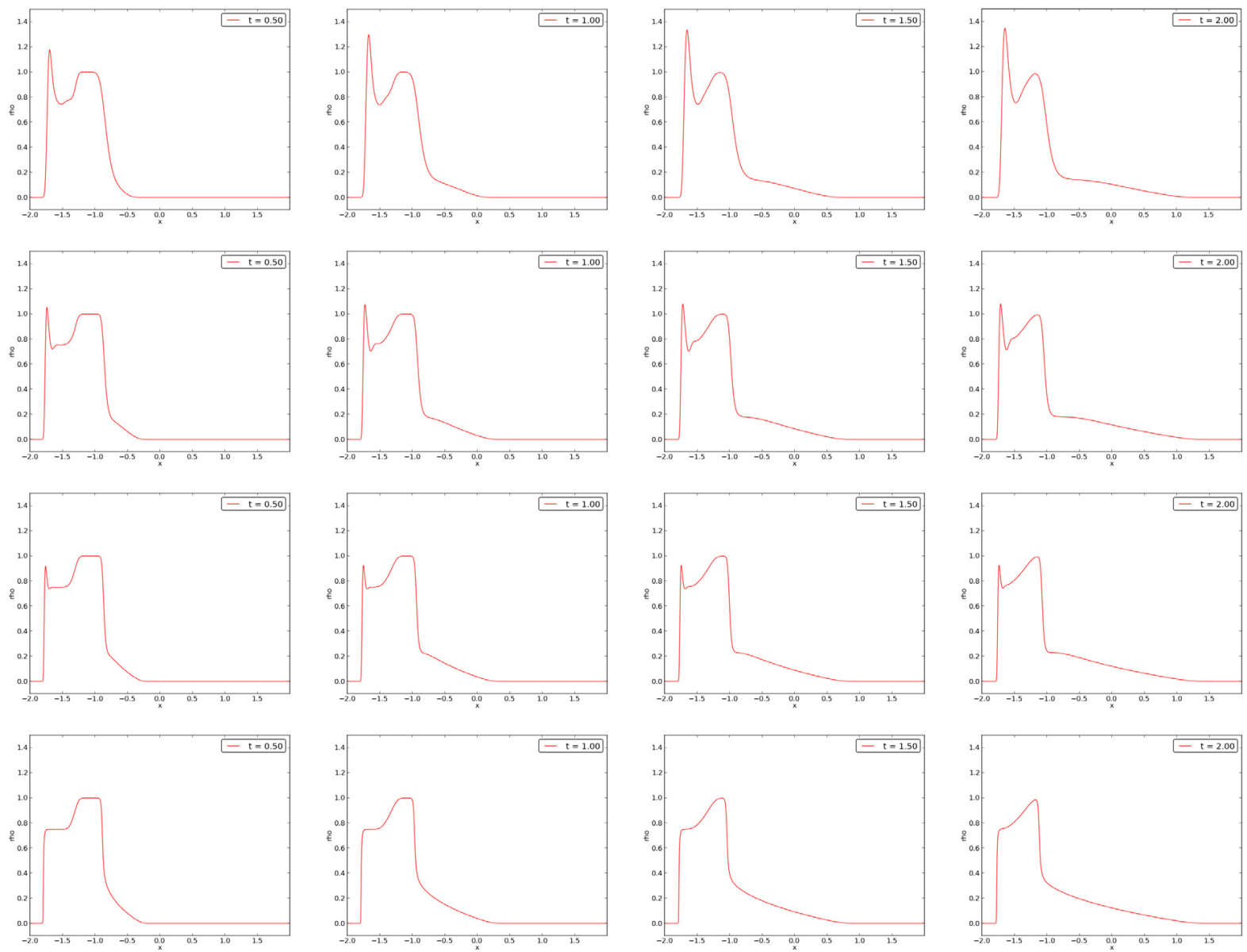

FIgURE 5. Integration of (1.1)-(3.6)-(3.7): first row $a=0.25$; second $a=0.1$, third $a=0.05$. Last row, integration of (1.2)-(3.6). The four columns display the times $t=0.5,1.0,1.5$ and 2.0. Mixed waves are due to the non-convex flow (3.6), see Figure 4. The space mesh size is 0.0004 .

\section{TECHNiCAL DETAILS}

For any $a, b \in \mathbb{R}$, we denote $I(a, b)=] a, b[\cup] b, a[$. We use below the following classical notations:

$$
D^{+} a_{j}=a_{j+1}-a_{j}, \quad D^{-} a_{j}=a_{j}-a_{j-1}, \quad D^{2} a_{j}=a_{j+1}-2 a_{j}+a_{j-1}=\left(D^{+}-D^{-}\right) a_{j}
$$

and recall the trivial identities

$$
\begin{gathered}
D^{+}\left(a_{j} b_{j}\right)=\left(D^{+} a_{j}\right) b_{j+1}+\left(D^{+} b_{j}\right) a_{j}, \quad D^{-}\left(a_{j} b_{j}\right)=\left(D^{-} a_{j}\right) b_{j}+\left(D^{-} b_{j}\right) a_{j-1}, \\
D^{2}\left(a_{j} b_{j}\right)=\left(D^{2} a_{j}\right) b_{j}+\left(D^{2} b_{j}\right) a_{j}+D^{+} a_{j} D^{+} b_{j}+D^{-} a_{j} D^{-} b_{j} .
\end{gathered}
$$

For later use, we note that the algorithm (2.4) can then be rewritten as

$$
\rho_{j}^{n+1}=\rho_{j}^{n}-\lambda \frac{D^{+}\left(f\left(t^{n}, x_{j-1 / 2}, \rho_{j}^{n}\right) v\left(c_{j-1 / 2}\right)\right)+D^{-}\left(f\left(t^{n}, x_{j+1 / 2}, \rho_{j}^{n}\right) v\left(c_{j+1 / 2}\right)\right)}{2}+\frac{1}{6} D^{2} \rho_{j}^{n} .
$$



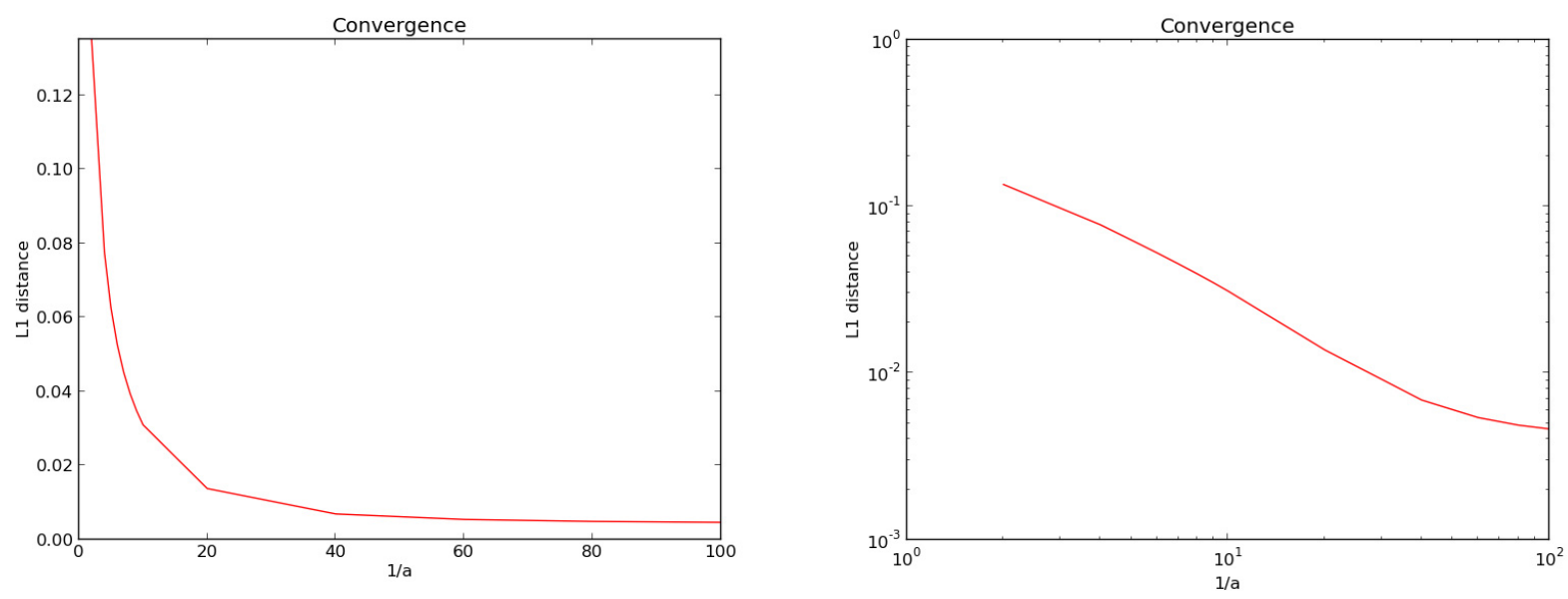

FiguRE 6. $\mathbf{L}^{1}$-distance between the solution $\rho_{a}$ to (1.1)-(3.6)-(3.7) and the solution $\rho$ to (1.2)(3.6) at time $t=0.500$, as a function of $1 / a$. Left, with a linear scale and, right, with a $\log$ scale.

Proof of Lemma 2.2. Note that, by (2.4), standard computations lead to

$$
\rho_{j}^{n+1}=\left(1-\alpha_{j}^{n}-\beta_{j}^{n}\right) \rho_{j}^{n}+\alpha_{j}^{n} \rho_{j-1}^{n}+\beta_{j}^{n} \rho_{j+1}^{n}-\lambda\left(\mathbf{f}_{j+1 / 2}^{n}\left(\rho_{j}^{n}, \rho_{j}^{n}\right)-\mathbf{f}_{j-1 / 2}^{n}\left(\rho_{j}^{n}, \rho_{j}^{n}\right)\right) .
$$

where

$$
\alpha_{j}^{n}=\lambda \frac{\mathbf{f}_{j-1 / 2}^{n}\left(\rho_{j}^{n}, \rho_{j}^{n}\right)-\mathbf{f}_{j-1 / 2}^{n}\left(\rho_{j-1}^{n}, \rho_{j}^{n}\right)}{\rho_{j}^{n}-\rho_{j-1}^{n}} \quad \text { and } \quad \beta_{j}^{n}=-\lambda \frac{\mathbf{f}_{j+1 / 2}^{n}\left(\rho_{j}^{n}, \rho_{j+1}^{n}\right)-\mathbf{f}_{j+1 / 2}^{n}\left(\rho_{j}^{n}, \rho_{j}^{n}\right)}{\rho_{j+1}^{n}-\rho_{j}^{n}}
$$

We now show that under condition (2.3), the following inequalities hold:

$$
\begin{aligned}
\alpha_{j}^{n} & \in[0,1 / 3] \\
\beta_{j}^{n} & \in[0,1 / 3] \quad \text { and } \quad \lambda\left(\mathbf{f}_{j+1 / 2}^{n}\left(\rho_{j}^{n}, \rho_{j}^{n}\right)-\mathbf{f}_{j-1 / 2}^{n}\left(\rho_{j}^{n}, \rho_{j}^{n}\right)\right) \leq \frac{1}{3} \rho_{j}^{n} . \\
1-\alpha_{j}^{n}-\beta_{j}^{n} & \in[1 / 3,1]
\end{aligned}
$$

Indeed,

$$
\begin{aligned}
\beta_{j}^{n} & =-\lambda \frac{\mathbf{f}_{j+1 / 2}^{n}\left(\rho_{j}^{n}, \rho_{j+1}^{n}\right)-\mathbf{f}_{j+1 / 2}^{n}\left(\rho_{j}^{n}, \rho_{j}^{n}\right)}{\rho_{j+1}^{n}-\rho_{j}^{n}} \\
& =-\frac{\lambda}{2}\left(\frac{f\left(t^{n}, x_{j+1 / 2}, \rho_{j+1}^{n}\right)-f\left(t^{n}, x_{j+1 / 2}, \rho_{j}^{n}\right)}{\rho_{j+1}^{n}-\rho_{j}^{n}} v\left(c_{j+1 / 2}^{n}\right)-\frac{1}{3 \lambda}\right) \\
& =-\frac{\lambda}{2} \partial_{\rho} f\left(t^{n}, x_{j+1 / 2}, \zeta_{j+1 / 2}\right) v\left(c_{j+1 / 2}^{n}\right)+\frac{1}{6},
\end{aligned}
$$

So that

$$
\begin{aligned}
& \beta_{j}^{n} \geq \frac{1}{2}\left(\frac{1}{3}-\lambda\left\|\partial_{\rho} f\right\|_{\mathbf{L}^{\infty}}\|v\|_{\mathbf{L}^{\infty}}\right) \geq 0 \\
& \beta_{j}^{n} \leq \frac{1}{2}\left(\frac{1}{3}+\lambda\left\|\partial_{\rho} f\right\|_{\mathbf{L}^{\infty}}\|v\|_{\mathbf{L}^{\infty}}\right) \leq \frac{1}{3} .
\end{aligned}
$$


Entirely similar computations lead to analogous estimates for $\alpha_{j}^{n}$. The bounds on $1-\alpha_{j}^{n}-\beta_{j}^{n}$ follow. The last term in (4.3), using (2.5) and (2.3), is estimated as follows

$$
\begin{aligned}
& \mathbf{f}_{j+1 / 2}^{n}\left(\rho_{j}^{n}, \rho_{j}^{n}\right)-\mathbf{f}_{j-1 / 2}^{n}\left(\rho_{j}^{n}, \rho_{j}^{n}\right) \\
\leq & \left|f\left(t^{n}, x_{j+1 / 2}, \rho_{j}^{n}\right) v\left(c_{j+1 / 2}^{n}\right)-f\left(t^{n}, x_{j-1 / 2}, \rho_{j}^{n}\right) v\left(c_{j-1 / 2}^{n}\right)\right| \\
\leq & \left|f\left(t^{n}, x_{j+1 / 2}, \rho_{j}^{n}\right)-f\left(t^{n}, x_{j-1 / 2}, \rho_{j}^{n}\right)\right| v\left(c_{j-1 / 2}^{n}\right)+\left|f\left(t^{n}, x_{j-1 / 2}, \rho_{j}^{n}\right)\right|\left|v\left(c_{j+1 / 2}^{n}\right)-v\left(c_{j-1 / 2}^{n}\right)\right| \\
\leq & h\left|\partial_{x} f\left(t^{n}, \zeta_{j}, \rho_{j}^{n}\right)\right|\|v\|_{\mathbf{L}^{\infty}}+2\left\|\partial_{\rho} f\right\|_{\mathbf{L}^{\infty}}\|v\|_{\mathbf{L}^{\infty}}\left|\rho_{j}^{n}\right| \\
\leq & \left(C\|v\|_{\mathbf{L}^{\infty}} h+2\left\|\partial_{\rho} f\right\|_{\mathbf{L}^{\infty}}\|v\|_{\mathbf{L}^{\infty}}\right)\left|\rho_{j}^{n}\right| \\
\leq & \left(C h+2\left\|\partial_{\rho} f\right\|_{\mathbf{L}^{\infty}}\right)\|v\|_{\mathbf{L}^{\infty}}\left|\rho_{j}^{n}\right| \\
\leq & \frac{1}{3 \lambda} \rho_{j}^{n} .
\end{aligned}
$$

Using the bounds (4.3) in (4.1), we obtain

$$
\rho_{j}^{n+1} \geq\left(1-\alpha_{j}^{n}-\beta_{j}^{n}\right) \rho_{j}^{n}+\alpha_{j}^{n} \rho_{j-1}^{n}+\beta_{j}^{n} \rho_{j+1}^{n}-\frac{1}{3} \rho_{j}^{n} \geq 0,
$$

proving the positivity of the discrete solution.

Proof of Lemma 2.4. Thanks to the positivity of the discrete solution, it is sufficient to compute

$$
\begin{aligned}
\left\|\rho^{n+1}\right\|_{\mathbf{L}^{1}} & =\sum_{j} h \rho_{j}^{n+1} \\
& =\sum_{j} h\left(\rho_{j}^{n}-\lambda\left(\mathbf{f}_{j+1 / 2}^{n}\left(\rho_{j}^{n}, \rho_{j+1}^{n}\right)-\mathbf{f}_{j-1 / 2}^{n}\left(\rho_{j-1}^{n}, \rho_{j}^{n}\right)\right)\right) \\
& =\sum_{j} h \rho_{j}^{n}-h \lambda\left(\lim _{i \rightarrow-\infty} \mathbf{f}_{i+1 / 2}^{n}\left(\rho_{i}^{n}, \rho_{i+1}^{n}\right)-\lim _{i \rightarrow+\infty} \mathbf{f}_{i-1 / 2}^{n}\left(\rho_{i-1}^{n}, \rho_{i}^{n}\right)\right) \\
& =\left\|\rho^{n}\right\|_{\mathbf{L}^{1}}
\end{aligned}
$$

completing the proof.

Proof of Lemma 2.5. For later use, estimate the quantity

$$
\begin{aligned}
\left|c_{j+1 / 2}^{n}-c_{j-1 / 2}^{n}\right| & \leq \sum_{k \in \mathbb{Z}} h\left|\rho_{k+1 / 2}^{n}\left(\eta_{k-(j+1 / 2)}-\eta_{k-(j-1 / 2)}\right)\right| \\
& \leq \sum_{k \in \mathbb{Z}} h \rho_{k+1 / 2}^{n} \int_{x_{k-j-1 / 2}}^{x_{k-j+1 / 2}}\left|\eta^{\prime}(s)\right| \mathrm{d} s \\
& \leq h\left\|\rho^{n}\right\|_{\mathbf{L}^{1}}\left\|\eta^{\prime}\right\|_{\mathbf{L}^{\infty}} \\
& =h\left\|\rho^{o}\right\|_{\mathbf{L}^{1}}\left\|\eta^{\prime}\right\|_{\mathbf{L}^{\infty}},
\end{aligned}
$$


where Lemma 2.4 was used. Using the same estimates as in the proof of Lemma 2.2, equality (4.1) yields

$$
\begin{aligned}
& \rho_{j}^{n+1} \leq\left(1-\alpha_{j}^{n}-\beta_{j}^{n}\right) \rho_{j}^{n}+\alpha_{j}^{n} \rho_{j-1}^{n}+\beta_{j}^{n} \rho_{j+1}^{n} \\
& +\lambda\left|f\left(t^{n}, x_{j+1 / 2}, \rho_{j}^{n}\right)-f\left(t^{n}, x_{j-1 / 2}, \rho_{j}^{n}\right)\right| v\left(c_{j-1 / 2}^{n}\right) \\
& +\lambda\left|f\left(t^{n}, x_{j-1 / 2}, \rho_{j}^{n}\right)\right|\left|D^{-} v\left(c_{j+1 / 2}^{n}\right)\right| \\
& \leq\left(1-\alpha_{j}^{n}-\beta_{j}^{n}\right)\left\|\rho^{n}\right\|_{\mathbf{L}_{\infty}}+\alpha_{j}^{n}\left\|\rho^{n}\right\|_{\mathbf{L}_{\infty}}+\beta_{j}^{n}\left\|\rho^{n}\right\|_{\mathbf{L}^{\infty}} \\
& +\lambda\left(h\left|\partial_{x} f\left(t^{n}, \zeta_{j}, \rho_{j}^{n}\right)\right|\|v\|_{\mathbf{L}^{\infty}}+\rho_{j}^{n}\left\|\partial_{\rho} f\right\|_{\mathbf{L}^{\infty}}\left\|v^{\prime}\right\|_{\mathbf{L}^{\infty}}\left|c_{j+1 / 2}^{n}-c_{j-1 / 2}^{n}\right|\right) \\
& \leq\left\|\rho^{n}\right\|_{\mathbf{L}^{\infty}}+\tau\left(C\|v\|_{\mathbf{L}^{\infty}}+\left\|\partial_{\rho} f\right\|_{\mathbf{L}^{\infty}}\left\|v^{\prime}\right\|_{\mathbf{L}^{\infty}}\left\|\rho^{o}\right\|_{\mathbf{L}^{1}}\left\|\eta^{\prime}\right\|_{\mathbf{L}^{\infty}}\right)\left\|\rho^{n}\right\|_{\mathbf{L}^{\infty}} \\
& \leq \mathrm{e}^{\mathcal{L} \tau}\left\|\rho^{n}\right\|_{\mathbf{L}^{\infty}}
\end{aligned}
$$

provided

$$
\mathcal{L}=C\|v\|_{\mathbf{L}^{\infty}}+\left\|\partial_{\rho} f\right\|_{\mathbf{L}^{\infty}}\left\|v^{\prime}\right\|_{\mathbf{L}^{\infty}}\left\|\rho^{o}\right\|_{\mathbf{L}^{1}}\left\|\eta^{\prime}\right\|_{\mathbf{L}^{\infty}} .
$$

A standard iterative argument completes the proof.

Proof of Proposition 2.6. First, we write (2.4) for $j$ and for $j+1$, subtract and get

$$
\rho_{j+1}^{n+1}-\rho_{j}^{n+1}=\rho_{j+1}^{n}-\rho_{j}^{n}-\lambda\left(\mathbf{f}_{j+3 / 2}^{n}\left(\rho_{j+1}^{n}, \rho_{j+2}^{n}\right)-\mathbf{f}_{j+1 / 2}^{n}\left(\rho_{j}^{n}, \rho_{j+1}^{n}\right)+\mathbf{f}_{j+1 / 2}^{n}\left(\rho_{j}^{n}, \rho_{j+1}^{n}\right)-\mathbf{f}_{j-1 / 2}^{n}\left(\rho_{j-1}^{n}, \rho_{j}^{n}\right)\right) .
$$

Now add and subtract $\mathbf{f}_{j+3 / 2}^{n}\left(\rho_{j}^{n}, \rho_{j+1}^{n}\right)+\mathbf{f}_{j+1 / 2}^{n}\left(\rho_{j-1}^{n}, \rho_{j}^{n}\right)$, then rearrange to obtain

$$
\rho_{j+1}^{n+1}-\rho_{j}^{n+1}=\mathcal{A}_{j}^{n}-\lambda \mathcal{B}_{j}^{n}
$$

where

$$
\begin{aligned}
\mathcal{A}_{j}^{n}= & \rho_{j+1}^{n}-\rho_{j}^{n} \\
& -\lambda\left(\mathbf{f}_{j+3 / 2}^{n}\left(\rho_{j+1}^{n}, \rho_{j+2}^{n}\right)-\mathbf{f}_{j+1 / 2}^{n}\left(\rho_{j}^{n}, \rho_{j+1}^{n}\right)-\mathbf{f}_{j+3 / 2}^{n}\left(\rho_{j}^{n}, \rho_{j+1}^{n}\right)+\mathbf{f}_{j+1 / 2}^{n}\left(\rho_{j-1}^{n}, \rho_{j}^{n}\right)\right) \\
\mathcal{B}_{j}^{n}= & \mathbf{f}_{j+3 / 2}^{n}\left(\rho_{j}^{n}, \rho_{j+1}^{n}\right)-\mathbf{f}_{j+1 / 2}^{n}\left(\rho_{j}^{n}, \rho_{j+1}^{n}\right)+\mathbf{f}_{j-1 / 2}^{n}\left(\rho_{j-1}^{n}, \rho_{j}^{n}\right)-\mathbf{f}_{j+1 / 2}^{n}\left(\rho_{j-1}^{n}, \rho_{j}^{n}\right) .
\end{aligned}
$$

Consider first the term $\mathcal{A}_{j}^{n}$. Recall (4.2) and observe that, after suitable rearrangements,

$$
\begin{aligned}
\mathcal{A}_{j}^{n}= & \left(\rho_{j+1}^{n}-\rho_{j}^{n}\right) \\
& \times\left[1+\lambda \frac{\mathbf{f}_{j+1 / 2}^{n}\left(\rho_{j}^{n}, \rho_{j+1}^{n}\right)-\mathbf{f}_{j+1 / 2}^{n}\left(\rho_{j}^{n}, \rho_{j}^{n}\right)}{\rho_{j+1}^{n}-\rho_{j}^{n}}-\lambda \frac{\mathbf{f}_{j+3 / 2}^{n}\left(\rho_{j+1}^{n}, \rho_{j+1}^{n}\right)-\mathbf{f}_{j+3 / 2}^{n}\left(\rho_{j}^{n}, \rho_{j+1}^{n}\right)}{\rho_{j+1}^{n}-\rho_{j}^{n}}\right] \\
& +\left(\rho_{j+2}^{n}-\rho_{j+1}^{n}\right)\left(-\lambda \frac{\mathbf{f}_{j+3 / 2}^{n}\left(\rho_{j+1}^{n}, \rho_{j+2}^{n}\right)-\mathbf{f}_{j+3 / 2}^{n}\left(\rho_{j+1}^{n}, \rho_{j+1}^{n}\right)}{\rho_{j+2}^{n}-\rho_{j+1}^{n}}\right) \\
& +\left(\rho_{j}^{n}-\rho_{j-1}^{n}\right)\left(\lambda \frac{\mathbf{f}_{j+1 / 2}^{n}\left(\rho_{j}^{n}, \rho_{j}^{n}\right)-\mathbf{f}_{j+1 / 2}^{n}\left(\rho_{j-1}^{n}, \rho_{j}^{n}\right)}{\rho_{j}^{n}-\rho_{j-1}^{n}}\right) \\
= & \left(1-\beta_{j}^{n}-\gamma_{j+1}^{n}\right)\left(\rho_{j+1}^{n}-\rho_{j}^{n}\right)+\beta_{j+1}^{n}\left(\rho_{j+2}^{n}-\rho_{j+1}^{n}\right)+\gamma_{j}^{n}\left(\rho_{j}^{n}-\rho_{j-1}^{n}\right)
\end{aligned}
$$

where $\gamma_{j}^{n}=\lambda\left(\mathbf{f}_{j+1 / 2}^{n}\left(\rho_{j}^{n}, \rho_{j}^{n}\right)-\mathbf{f}_{j+1 / 2}^{n}\left(\rho_{j-1}^{n}, \rho_{j}^{n}\right)\right) /\left(\rho_{j}^{n}-\rho_{j-1}^{n}\right)$ and the bounds $\gamma_{j}^{n} \in[0,1 / 3]$ can be proved exactly as was done with $\alpha_{j}^{n}$, thanks to the CFL condition (2.3). By convexity,

$$
\sum_{j \in \mathbb{Z}}\left|\mathcal{A}_{n}^{j}\right| \leq \sum_{j \in \mathbb{Z}}\left|\rho_{j+1}^{n}-\rho_{j}^{n}\right|
$$


We now turn to the term $\mathcal{B}_{j}^{n}$ in (4.6). Since

$$
\begin{aligned}
\mathcal{B}_{j}^{n}= & \frac{f\left(t^{n}, x_{j+3 / 2}, \rho_{j}^{n}\right) v\left(c_{j+3 / 2}\right)-2 f\left(t^{n}, x_{j+1 / 2}, \rho_{j}^{n}\right) v\left(c_{j+1 / 2}\right)+f\left(t^{n}, x_{j-1 / 2}, \rho_{j}^{n}\right) v\left(c_{j-1 / 2}\right)}{2} \\
& +\frac{f\left(t^{n}, x_{j+3 / 2}, \rho_{j+1}^{n}\right) v\left(c_{j+3 / 2}\right)-f\left(t^{n}, x_{j+1 / 2}, \rho_{j+1}^{n}\right) v\left(c_{j+1 / 2}\right)}{2} \\
& -\frac{f\left(t^{n}, x_{j+1 / 2}, \rho_{j-1}^{n}\right) v\left(c_{j+1 / 2}\right)-f\left(t^{n}, x_{j-1 / 2}, \rho_{j-1}^{n}\right) v\left(c_{j-1 / 2}\right)}{2},
\end{aligned}
$$

we consider the various terms separately.

$$
\begin{aligned}
(4.9)= & v\left(c_{j+1 / 2}\right) \frac{f\left(t^{n}, x_{j+3 / 2}, \rho_{j}^{n}\right)-2 f\left(t^{n}, x_{j+1 / 2}, \rho_{j}^{n}\right)+f\left(t^{n}, x_{j-1 / 2}, \rho_{j}^{n}\right)}{2} \\
& +f\left(t^{n}, x_{j+1 / 2}, \rho_{j}^{n}\right) \frac{v\left(c_{j+3 / 2}\right)-2 v\left(c_{j+1 / 2}\right)+v\left(c_{j-1 / 2}\right)}{2} \\
& +\frac{f\left(t^{n}, x_{j+3 / 2}, \rho_{j}^{n}\right)-f\left(t^{n}, x_{j+1 / 2}, \rho_{j}^{n}\right)}{2} \frac{v\left(c_{j+3 / 2}\right)-v\left(c_{j+1 / 2}\right)}{2} \\
& +\frac{f\left(t^{n}, x_{j+1 / 2}, \rho_{j}^{n}\right)-f\left(t^{n}, x_{j-1 / 2}, \rho_{j}^{n}\right)}{2} \frac{v\left(c_{j+1 / 2}\right)-v\left(c_{j-1 / 2}\right)}{2}
\end{aligned}
$$

where

$$
\begin{aligned}
& \left|\frac{f\left(t^{n}, x_{j+3 / 2}, \rho_{j}^{n}\right)-2 f\left(t^{n}, x_{j+1 / 2}, \rho_{j}^{n}\right)+f\left(t^{n}, x_{j-1 / 2}, \rho_{j}^{n}\right)}{2}\right| \\
= & \left|\frac{f\left(t^{n}, x_{j+3 / 2}, \rho_{j}^{n}\right)-f\left(t^{n}, x_{j+1 / 2}, \rho_{j}^{n}\right)}{2}-\frac{f\left(t^{n}, x_{j+1 / 2}, \rho_{j}^{n}\right)+f\left(t^{n}, x_{j-1 / 2}, \rho_{j}^{n}\right)}{2}\right| \\
\leq & \frac{h}{2}\left|\partial_{x} f\left(t^{n}, \zeta_{j+1}, \rho_{j}^{n}\right)-\partial_{x} f\left(t^{n}, \zeta_{j-1}, \rho_{j}^{n}\right)\right| \\
\leq & \frac{h}{2} \int_{\zeta_{j-1}}^{\zeta_{j+1}}\left|\partial_{x x}^{2} f\left(t_{x}, x, \rho_{j}^{n}\right)\right| \mathrm{d} x \\
\leq & C h^{2}\left|\rho_{j}^{n}\right|
\end{aligned}
$$

where (2.1) was used to get to the last line. Moreover,

$$
\begin{aligned}
& \frac{v\left(c_{j+3 / 2}\right)-2 v\left(c_{j+1 / 2}\right)+v\left(c_{j-1 / 2}\right)}{2} \\
= & \frac{v\left(c_{j+3 / 2}\right)-v\left(c_{j+1 / 2}\right)}{2}-\frac{v\left(c_{j+1 / 2}\right)+v\left(c_{j-1 / 2}\right)}{2} \\
= & \frac{1}{2}\left(v^{\prime}\left(\zeta_{j}\right)\left(c_{j+1 / 2}^{n}-c_{j-1 / 2}^{n}\right)-v^{\prime}\left(\zeta_{j+1}\right)\left(c_{j+3 / 2}^{n}-c_{j+1 / 2}^{n}\right)\right) \\
= & \frac{1}{2}\left(v^{\prime}\left(\zeta_{j}\right)-v^{\prime}\left(\zeta_{j+1}\right)\right)\left(c_{j+1 / 2}^{n}-c_{j-1 / 2}^{n}\right)-\frac{1}{2} v^{\prime}\left(\zeta_{j+1}\right)\left(c_{j+3 / 2}^{n}+2 c_{j+1 / 2}^{n}-c_{j-1 / 2}^{n}\right) \\
= & \frac{1}{2} v^{\prime \prime}\left(\xi_{j}\right)\left(\zeta_{j}-\zeta_{j+1}\right)\left(c_{j+1 / 2}^{n}-c_{j-1 / 2}^{n}\right)-\frac{1}{2} v^{\prime}\left(\zeta_{j+1}\right)\left(c_{j+3 / 2}^{n}+2 c_{j+1 / 2}^{n}-c_{j-1 / 2}^{n}\right) .
\end{aligned}
$$

Note that we have $\left|\zeta_{j}-\zeta_{j+1}\right| \leq\left|c_{j+3 / 2}^{n}-c_{j+1 / 2}^{n}\right|+\left|c_{j+1 / 2}^{n}-c_{j-1 / 2}^{n}\right|$, and so using Young's inequality,

$$
\begin{aligned}
\left|\frac{v\left(c_{j+3 / 2}\right)-2 v\left(c_{j+1 / 2}\right)+v\left(c_{j-1 / 2}\right)}{2}\right| \leq & \frac{1}{2}\left\|v^{\prime \prime}\right\|_{\mathbf{L}^{\infty}}\left[\frac{3}{2}\left|c_{j+1 / 2}^{n}-c_{j-1 / 2}^{n}\right|^{2}+\frac{1}{2}\left|c_{j+3 / 2}^{n}-c_{j+1 / 2}^{n}\right|^{2}\right] \\
& +\frac{1}{2}\left\|v^{\prime}\right\|_{\mathbf{L}^{\infty}}\left|c_{j+3 / 2}^{n}+2 c_{j+1 / 2}^{n}-c_{j-1 / 2}^{n}\right| .
\end{aligned}
$$


We now estimate the terms involving the discrete derivatives of $c_{j}^{n}$ in the expression above, exploiting the regularity (2.2) of $\eta$. By (2.6), we have

$$
\begin{aligned}
\left|c_{j+1 / 2}^{n}-c_{j-1 / 2}^{n}\right| & =\left|\sum_{k \in \mathbb{Z}} h \rho_{k+1 / 2}^{n}\left(\eta_{k-(j+1 / 2)}-\eta_{k-(j-1 / 2)}\right)\right| \\
& \leq \sum_{k \in \mathbb{Z}} h\left|\rho_{k-j-1 / 2}^{n}\right|\left|\eta_{k+1 / 2}-\eta_{k-1 / 2}\right| \\
& \leq \sum_{k \in \mathbb{Z}} h\left|\rho_{k-j-1 / 2}^{n}\right| \int_{x_{k-1 / 2}}^{x_{k+1 / 2}}\left|\eta^{\prime}(s)\right| \mathrm{d} s \\
& \leq h\left\|\rho^{n}\right\|_{\mathbf{L}^{1}}\left\|\eta^{\prime}\right\|_{\mathbf{L}^{\infty}} .
\end{aligned}
$$

Similarly,

$$
\begin{aligned}
\left|c_{j+3 / 2}^{n}+2 c_{j+1 / 2}^{n}-c_{j-1 / 2}^{n}\right| & \leq \sum_{k \in \mathbb{Z}} h\left|\rho_{k-j-1 / 2}^{n}\right|\left|\eta_{k-1 / 2}-2 \eta_{k+1 / 2}+\eta_{k+3 / 2}\right| \\
& \leq h \sum_{k \in \mathbb{Z}} h\left|\rho_{k-j-1 / 2}^{n}\right|\left|\eta^{\prime}\left(\zeta_{k+1}\right)-\eta^{\prime}\left(\zeta_{k}\right)\right| \\
& =h \sum_{k \in \mathbb{Z}} h\left|\rho_{k-j-1 / 2}^{n}\right| \int_{\zeta_{k}}^{\zeta_{k+1}}\left|\eta^{\prime \prime}(s)\right| \mathrm{d} s \\
& \leq h \sum_{k \in \mathbb{Z}} h\left|\rho_{k-j-1 / 2}^{n}\right| \int_{x_{k-1 / 2}}^{x_{k+3 / 2}}\left|\eta^{\prime \prime}(s)\right| \mathrm{d} s \\
& =2 h^{2}\left\|\rho^{n}\right\|_{\mathbf{L}^{1}}\left\|\eta^{\prime \prime}\right\|_{\mathbf{L}^{\infty}},
\end{aligned}
$$

to complete the estimate of (4.9) we use the results above to bound the remaining terms

$$
\begin{aligned}
\left|\frac{f\left(t^{n}, x_{j+3 / 2}, \rho_{j}^{n}\right)-f\left(t^{n}, x_{j+1 / 2}, \rho_{j}^{n}\right)}{2}\right| & \leq \frac{1}{2} h C\left|\rho_{j}^{n}\right| \\
\left|\frac{v\left(c_{j+3 / 2}\right)-v\left(c_{j+1 / 2}\right)}{2}\right| & \leq \frac{1}{2} h\left\|v^{\prime}\right\|_{\mathbf{L}^{\infty}}\left\|\eta^{\prime}\right\|_{\mathbf{L}^{\infty}}\left\|\rho^{o}\right\|_{\mathbf{L}^{1}} \\
\left|\frac{f\left(t^{n}, x_{j+1 / 2}, \rho_{j}^{n}\right)-f\left(t^{n}, x_{j-1 / 2}, \rho_{j}^{n}\right)}{2}\right| & \leq \frac{1}{2} h C\left|\rho_{j}^{n}\right| \\
\left|\frac{v\left(c_{j+1 / 2}\right)-v\left(c_{j-1 / 2}\right)}{2}\right| & \leq \frac{1}{2} h\left\|v^{\prime}\right\|_{\mathbf{L}^{\infty}}\left\|\eta^{\prime}\right\|_{\mathbf{L}^{\infty}}\left\|\rho^{o}\right\|_{\mathbf{L}^{1}}
\end{aligned}
$$

and we are now able to complete the estimate of (4.9):

$$
\begin{aligned}
(4.9) \leq & h^{2} C\|v\|_{\mathbf{L}^{\infty}}\left|\rho_{j}^{n}\right| \\
& +\left\|\partial_{\rho} f\right\|_{\mathbf{L}^{\infty}}\left|\rho_{j}^{n}\right|\left(h^{2}\left\|v^{\prime \prime}\right\|_{\mathbf{L}^{\infty}}\left\|\rho^{o}\right\|_{\mathbf{L}^{1}}^{2}\left\|\eta^{\prime}\right\|_{\mathbf{L}^{\infty}}+h^{2}\left\|v^{\prime}\right\|_{\mathbf{L}^{\infty}}\left\|\rho^{o}\right\|_{\mathbf{L}^{1}}^{2}\left\|\eta^{\prime \prime}\right\|_{\mathbf{L}^{\infty}}\right) \\
& +\frac{1}{2} h^{2} C\left\|v^{\prime}\right\|_{\mathbf{L}^{\infty}}\left\|\eta^{\prime}\right\|_{\mathbf{L}^{\infty}}\left\|\rho^{o}\right\|_{\mathbf{L}^{1}}\left|\rho_{j}^{n}\right| \\
= & h^{2}\left[C\|v\|_{\mathbf{L}^{\infty}}+\left(\left\|\partial_{\rho} f\right\|_{\mathbf{L}^{\infty}}\left(\left\|v^{\prime \prime}\right\|_{\mathbf{L}^{\infty}}\left\|\eta^{\prime}\right\|_{\mathbf{L}^{\infty}}+\left\|v^{\prime}\right\|_{\mathbf{L}^{\infty}}\left\|\eta^{\prime \prime}\right\|_{\mathbf{L}^{\infty}}\right)\right.\right. \\
& \left.\left.+\frac{C}{2}\left\|v^{\prime}\right\|_{\mathbf{L}^{\infty}}\left\|\eta^{\prime}\right\|_{\mathbf{L}^{\infty}}\right)\left\|\rho^{o}\right\|_{\mathbf{L}^{1}}\right]\left|\rho_{j}^{n}\right| \\
\leq & h^{2}\|v\|_{\mathbf{W}^{2, \infty}}\left(C+\left(\left\|\partial_{\rho} f\right\|_{\mathbf{L}^{\infty}}+\frac{C}{2}\right)\left\|\eta^{\prime}\right\|_{\mathbf{W}^{1, \infty}}\left\|\rho^{o}\right\|_{\mathbf{L}^{1}}\right)\left|\rho_{j}^{n}\right|
\end{aligned}
$$


We now pass to estimate (4.10) and (4.11):

$$
\begin{aligned}
(4.10)+(4.11)= & \frac{1}{2}\left(f\left(t^{n}, x_{j+3 / 2}, \rho_{j+1}^{n}\right)-f\left(t^{n}, x_{j+1 / 2}, \rho_{j+1}^{n}\right)\right) v\left(c_{j+3 / 2}\right) \\
& +\frac{1}{2} f\left(t^{n}, x_{j+1 / 2}, \rho_{j+1}^{n}\right)\left(v\left(c_{j+3 / 2}\right)-v\left(c_{j+1 / 2}\right)\right) \\
& -\frac{1}{2}\left(f\left(t^{n}, x_{j+1 / 2}, \rho_{j-1}^{n}\right)-f\left(t^{n}, x_{j-1 / 2}, \rho_{j-1}^{n}\right)\right) v\left(c_{j+1 / 2}\right) \\
& -\frac{1}{2} f\left(t^{n}, x_{j-1 / 2}, \rho_{j-1}^{n}\right)\left(v\left(c_{j+1 / 2}\right)-v\left(c_{j-1 / 2}\right)\right) \\
= & \frac{1}{2} h\left(\partial_{x} f\left(t^{n}, \xi_{j+1}, \rho_{j+1}^{n}\right) v\left(c_{j+3 / 2}\right)-\partial_{x} f\left(t^{n}, \xi_{j}, \rho_{j-1}^{n}\right) v\left(c_{j+1 / 2}\right)\right) \\
& +\frac{1}{2}\left(f\left(t^{n}, x_{j+1 / 2}, \rho_{j+1}^{n}\right) v^{\prime}\left(\gamma_{j+1}\right)\left(c_{j+3 / 2}-c_{j+1 / 2}\right)\right. \\
& \left.-f\left(t^{n}, x_{j-1 / 2}, \rho_{j-1}^{n}\right) v^{\prime}\left(\gamma_{j}\right)\left(c_{j+1 / 2}-c_{j-1 / 2}\right)\right)
\end{aligned}
$$

for suitable $\left.\xi_{j} \in\right] x_{j-1 / 2}, x_{j+1 / 2}\left[\right.$ and $\gamma_{j} \in I\left(c_{j-1 / 2}, c_{j+1 / 2}\right)$. Introducing $\left.\hat{\xi}_{j} \in\right] \xi_{j}, \xi_{j+1}\left[, \hat{\zeta}_{j} \in I\left(\rho_{j-1}^{n}, \rho_{j+1}^{n}\right), \hat{\gamma}_{j} \in\right.$ $\left.I\left(\gamma_{j}, \gamma_{j+1}\right), \check{\xi}_{j} \in\right] x_{j-1 / 2}, x_{j+1 / 2}\left[, \check{\zeta}_{j} \in I\left(\rho_{j-1}^{n}, \rho_{j+1}^{n}\right), \check{\gamma}_{j} \in I\left(\gamma_{j}, \gamma_{j+1}\right), \delta_{j} \in I\left(c_{j+3 / 2}-c_{j+1 / 2}, c_{j+1 / 2}-c_{j-1 / 2}\right)\right.$ and using (2.1), (4.12), (4.13)

$$
\begin{aligned}
|(4.10)+(4.11)| \leq & \frac{1}{2} h\left(\left|\partial_{x x}^{2} f\left(t^{n}, \hat{\xi}_{j}, \hat{\zeta}_{j}\right)\right| v\left(\hat{\gamma}_{j+1}\right) h+\left|\partial_{\rho x}^{2} f\left(t^{n}, \hat{\xi}_{j}, \hat{\zeta}_{j}\right)\right| v\left(\hat{\gamma}_{j+1}\right)\left|\rho_{j+1}^{n}-\rho_{j-1}^{n}\right|\right. \\
& \left.+\left|\partial_{x} f\left(t^{n}, \hat{\zeta}_{j}, \hat{\zeta}_{j}\right)\right|\left|v^{\prime}\left(\hat{\gamma}_{j+1}\right)\right|\left|c_{j+3 / 2}-c_{j+1 / 2}\right|\right) \\
& +\frac{1}{2}\left(\left|\partial_{x} f\left(t^{n}, \check{\zeta}_{j}, \check{\zeta}_{j}\right)\right|\left|v^{\prime}\left(\check{\gamma}_{j}\right)\right|\left|\delta_{j}\right| h+\left|f\left(t^{n}, \check{\xi}_{j}, \check{\zeta}_{j}\right)\right|\left|v^{\prime \prime}\left(\check{\gamma}_{j}\right)\right|\left|\delta_{j}\right|\left|\gamma_{j+1}-\gamma_{j}\right|\right. \\
& \left.+\left|f\left(t^{n}, \check{\xi}_{j}, \check{\zeta}_{j}\right)\right|\left|v^{\prime}\left(\check{\gamma}_{j}\right)\right|\left|c_{j+3 / 2}-2 c_{j+1 / 2}-c_{j-1 / 2}\right|\right) \\
\leq & \frac{1}{2} h\left(C\|v\|_{\mathbf{L}^{\infty}}\left|\hat{\zeta}_{j}\right| h+\left\|\partial_{\rho x}^{2} f\right\|_{\mathbf{L}^{\infty}}\|v\|_{\mathbf{L}^{\infty}}\left|\rho_{j+1}^{n}-\rho_{j-1}^{n}\right|+C\left\|v^{\prime}\right\|_{\mathbf{L}^{\infty}}\left\|\rho^{o}\right\|_{\mathbf{L}^{1}}\left\|\eta^{\prime}\right\|_{\mathbf{L}^{\infty}}\left|\hat{\zeta}_{j}\right| h\right) \\
& +\frac{1}{2}\left(C\left\|v^{\prime}\right\|_{\mathbf{L}^{\infty}}\left\|\rho^{o}\right\|_{\mathbf{L}^{1}}\left\|\eta^{\prime}\right\|_{\mathbf{L}^{\infty}}\left|\check{\zeta}_{j}\right| h^{2}+\left\|\partial_{\rho} f\right\|_{\mathbf{L}^{\infty}}\left\|v^{\prime \prime}\right\|_{\mathbf{L}^{\infty}}\left\|\rho^{o}\right\|_{\mathbf{L}^{1}}^{2}\left\|\eta^{\prime}\right\|_{\mathbf{L}^{\infty}}^{2}\left|\check{\zeta}_{j}\right| h^{2}\right. \\
& \left.+2\left\|\partial_{\rho} f\right\|_{\mathbf{L}^{\infty}}\left\|v^{\prime}\right\|_{\mathbf{L}^{\infty}}\left\|\rho^{o}\right\|_{\mathbf{L}^{1}}\left\|\eta^{\prime \prime}\right\|_{\mathbf{L}^{\infty}}\left|\check{\zeta}_{j}\right| h^{2}\right) \\
= & \frac{1}{2} C\left(1+\left\|\eta^{\prime}\right\|_{\mathbf{L}^{\infty}}\left\|\rho^{o}\right\|_{\mathbf{L}^{1}}\right)\|v\|_{\mathbf{W}^{1, \infty}}\left|\hat{\zeta}_{j}\right| h^{2} \\
& +\frac{1}{2}\left(C+\left\|\partial_{\rho} f\right\|_{\mathbf{L}^{\infty}}\left\|\rho^{o}\right\|_{\mathbf{L}^{1}}\left\|\eta^{\prime}\right\|_{\mathbf{L}^{\infty}}+2\left\|\partial_{\rho} f\right\|_{\mathbf{L}^{\infty}}\right)\left\|v^{\prime}\right\|_{\mathbf{W}^{1, \infty}}\left\|\eta^{\prime}\right\|_{\mathbf{W}^{1, \infty}}\left\|\rho^{o}\right\|_{\mathbf{L}^{1}}\left|\check{\zeta}_{j}\right| h^{2} \\
& +\frac{1}{2}\left\|\partial_{\rho x}^{2} f\right\|_{\mathbf{L}^{\infty}}\|v\|_{\mathbf{L}^{\infty}}\left|\rho_{j+1}^{n}-\rho_{j-1}^{n}\right| h .
\end{aligned}
$$

The above bound allows to obtain the estimate for $\left|\mathcal{B}_{j}\right|$ :

$$
\begin{aligned}
\left|\mathcal{B}_{j}\right| \leq & \|v\|_{\mathbf{W}^{2, \infty}}\left(C+\left(\left\|\partial_{\rho} f\right\|_{\mathbf{L}^{\infty}}+\frac{C}{2}\right)\left\|\eta^{\prime}\right\|_{\mathbf{W}^{1, \infty}}\left\|\rho^{o}\right\|_{\mathbf{L}^{1}}\right)\left|\rho_{j}^{n}\right| h^{2} \\
& +\frac{1}{2} C\left(1+\left\|\eta^{\prime}\right\|_{\mathbf{L}^{\infty}}\left\|\rho^{o}\right\|_{\mathbf{L}^{1}}\right)\|v\|_{\mathbf{W}^{1, \infty}}\left|\hat{\zeta}_{j}\right| h^{2} \\
& +\frac{1}{2}\left(C+\left\|\partial_{\rho} f\right\|_{\mathbf{L}^{\infty}}\left\|\rho^{o}\right\|_{\mathbf{L}^{1}}\left\|\eta^{\prime}\right\|_{\mathbf{L}^{\infty}}+2\left\|\partial_{\rho} f\right\|_{\mathbf{L}^{\infty}}\right)\left\|v^{\prime}\right\|_{\mathbf{W}^{1, \infty}}\left\|\eta^{\prime}\right\|_{\mathbf{W}^{1, \infty}}\left\|\rho^{o}\right\|_{\mathbf{L}^{1}}\left|\check{\zeta}_{j}\right| h^{2} \\
& +\frac{1}{2}\left\|\partial_{\rho x}^{2} f\right\|_{\mathbf{L}^{\infty}}\|v\|_{\mathbf{L}^{\infty}}\left|\rho_{j+1}^{n}-\rho_{j-1}^{n}\right| h .
\end{aligned}
$$


so that

$$
\begin{aligned}
\sum_{j \in \mathbb{Z}}\left|\mathcal{B}_{j}\right| \leq & \|v\|_{\mathbf{W}^{2, \infty}}\left(C+\left(\left\|\partial_{\rho} f\right\|_{\mathbf{L}^{\infty}}+\frac{C}{2}\right)\left\|\eta^{\prime}\right\|_{\mathbf{W}^{1, \infty}}\left\|\rho^{o}\right\|_{\mathbf{L}^{1}}\right)\left\|\rho^{o}\right\|_{\mathbf{L}^{1}} h \\
& +\frac{1}{2} C\left(1+\left\|\eta^{\prime}\right\|_{\mathbf{L}^{\infty}}\left\|\rho^{o}\right\|_{\mathbf{L}^{1}}\right)\|v\|_{\mathbf{W}^{1, \infty}}\left\|\rho^{o}\right\|_{\mathbf{L}^{1}} h \\
& +\frac{1}{2}\left(C+\left\|\partial_{\rho} f\right\|_{\mathbf{L}^{\infty}}\left(2+\left\|\rho^{o}\right\|_{\mathbf{L}^{1}}\left\|\eta^{\prime}\right\|_{\mathbf{L}^{\infty}}\right)\right)\left\|v^{\prime}\right\|_{\mathbf{W}^{1, \infty}}\left\|\eta^{\prime}\right\|_{\mathbf{W}^{1, \infty}}\left\|\rho^{o}\right\|_{\mathbf{L}^{1}} h \\
& +\frac{1}{2}\left\|\partial_{\rho x}^{2} f\right\|_{\mathbf{L}^{\infty}}\|v\|_{\mathbf{L}^{\infty}}\left(\sum_{j \in \mathbb{Z}}\left|\rho_{j+1}^{n}-\rho_{j-1}^{n}\right|\right) h .
\end{aligned}
$$

Recall now (4.5) and (4.8) to obtain

$$
\sum_{j \in \mathbb{Z}}\left|\rho_{j+1}^{n+1}-\rho_{j}^{n+1}\right| \leq \sum_{j \in \mathbb{Z}}\left|\mathcal{A}_{j}\right|+\lambda \sum_{j \in \mathbb{Z}}\left|\mathcal{B}_{j}\right| \leq\left(1+\mathcal{K}_{1} \tau\right) \sum_{j \in \mathbb{Z}}\left|\rho_{j+1}^{n}-\rho_{j}^{n}\right|+\mathcal{K}_{2} \tau
$$

where

$$
\begin{aligned}
\mathcal{K}_{1}= & \left\|\partial_{\rho x}^{2} f\right\|_{\mathbf{L}^{\infty}}\|v\|_{\mathbf{L}^{\infty}} \\
\mathcal{K}_{2}= & {\left[\frac{3}{2} C+\left(\left\|\partial_{\rho} f\right\|_{\mathbf{L}^{\infty}}+C\right)\left\|\eta^{\prime}\right\|_{\mathbf{W}^{1, \infty}}\left\|\rho^{o}\right\|_{\mathbf{L}^{1}}\right.} \\
& \left.+\frac{1}{2}\left(C+\left\|\partial_{\rho} f\right\|_{\mathbf{L}^{\infty}}\left(2+\left\|\rho^{o}\right\|_{\mathbf{L}^{1}}\left\|\eta^{\prime}\right\|_{\mathbf{L}^{\infty}}\right)\right)\left\|\eta^{\prime}\right\|_{\mathbf{W}^{1, \infty}}\right]\|v\|_{\mathbf{W}^{2, \infty}}\left\|\rho^{o}\right\|_{\mathbf{L}^{1}} .
\end{aligned}
$$

The estimate (2.7) now follows from standard iterative procedure. The proof of Proposition 2.6 follows immediately.

Proof of Lemma 2.7. We follow the same line as in Section 3 of [17]. Using (4.12), Lemma 2.2, Lemma 2.4 and Proposition 2.6 compute preliminarily

$$
\begin{aligned}
& \sum_{j \in \mathbb{Z}}\left|D^{+}\left(f\left(t^{n}, x_{j-1 / 2}, \rho_{j}^{n}\right) v\left(c_{j-1 / 2}^{n}\right)\right)\right| \\
\leq & \sum_{j \in \mathbb{Z}}\left[\left|\partial_{x} f\left(t^{n}, \xi_{j}, \zeta_{j}\right) v\left(\gamma_{j}\right)\right| h+\left|\partial_{\rho} f\left(t^{n}, \xi_{j}, \zeta_{j}\right) v\left(\gamma_{j}\right)\right|\left|\rho_{j+1}^{n}-\rho_{j}^{n}\right|\right. \\
& \left.+\left|f\left(t^{n}, \xi_{j}, \zeta_{j}\right) v^{\prime}\left(\gamma_{j}\right)\right|\left|c_{j+1 / 2}^{n}-c_{j-1 / 2}^{n}\right|\right] \\
\leq & \sum_{j \in \mathbb{Z}}\left[C\|v\|_{\mathbf{L}^{\infty}} \rho_{j}^{n} h+\left\|\partial_{\rho} f\right\|_{\mathbf{L}^{\infty}}\|v\|_{\mathbf{L}^{\infty}}\left|\rho_{j+1}^{n}-\rho_{j}^{n}\right|\right. \\
& \left.+\left\|\partial_{\rho} f\right\|_{\mathbf{L}^{\infty}}\left\|v^{\prime}\right\|_{\mathbf{L}^{\infty}}\left\|\eta^{\prime}\right\|_{\mathbf{L}^{\infty}}\left\|\rho^{o}\right\|_{\mathbf{L}^{1}} \max \left\{\rho_{j}^{n}, \rho_{j+1}^{n}\right\} h\right] \\
\leq & C\|v\|_{\mathbf{L}^{\infty}}\left\|\rho^{o}\right\|_{\mathbf{L}^{1}}+2\left\|\partial_{\rho} f\right\|_{\mathbf{L}^{\infty}}\left\|\rho^{o}\right\|_{\mathbf{L}^{1}}^{2}\left\|\eta^{\prime}\right\|_{\mathbf{L}^{\infty}}\left\|v^{\prime}\right\|_{\mathbf{L}^{\infty}}+\left\|\partial_{\rho} f\right\|_{\mathbf{L}^{\infty}}\|v\|_{\mathbf{L}^{\infty}} \sum_{j \in \mathbb{Z}}\left|\rho_{j+1}^{n}-\rho_{j}^{n}\right| \\
\leq & C\|v\|_{\mathbf{L}^{\infty}}\left\|\rho^{o}\right\|_{\mathbf{L}^{1}}+2\left\|\partial_{\rho} f\right\|_{\mathbf{L}^{\infty}}\left\|\rho^{o}\right\|_{\mathbf{L}^{1}}^{2}\left\|\eta^{\prime}\right\|_{\mathbf{L}^{\infty}}\left\|v^{\prime}\right\|_{\mathbf{L}^{\infty}} \\
& +\left\|\partial_{\rho} f\right\|_{\mathbf{L}^{\infty}}\|v\|_{\mathbf{L}^{\infty}}\left(\mathcal{K}_{2} t+\sum_{j \in \mathbb{Z}}\left|\rho_{j+1}^{o}-\rho_{j}^{o}\right|\right) \mathrm{e}^{\mathcal{K}_{1} t} .
\end{aligned}
$$


The term $\sum_{j \in \mathbb{Z}}\left|D^{-}\left(f\left(t^{n}, x_{j+1 / 2}, \rho_{j}^{n}\right) v\left(c_{j+1 / 2}^{n}\right)\right)\right|$ admits an analogous estimate. Moreover,

$$
\sum_{j \in \mathbb{Z}}\left|D^{2} \rho_{j}^{n}\right| \leq 2 \sum_{j \in \mathbb{Z}}\left|\rho_{j+1}^{n}-\rho_{j}^{n}\right| \leq 2\left(\mathcal{K}_{2} t+\sum_{j \in \mathbb{Z}}\left|\rho_{j+1}^{o}-\rho_{j}^{o}\right|\right) \mathrm{e}^{\mathcal{K}_{1} t}
$$

Using the above estimates and (2.4) we get

$$
\begin{aligned}
\left\|\rho^{n+1}-\rho^{n}\right\|_{\mathbf{L}^{1}}= & \sum_{j \in \mathbb{Z}} h\left|\rho_{j}^{n+1}-\rho_{j}^{n}\right| \\
\leq & \frac{\tau}{2} \sum_{j \in \mathbb{Z}}\left|D^{+}\left(f\left(t^{n}, x_{j-1 / 2}, \rho_{j}^{n}\right) v\left(c_{j-1 / 2}^{n}\right)\right)\right| \\
& +\frac{\tau}{2} \sum_{j \in \mathbb{Z}}\left|D^{-}\left(f\left(t^{n}, x_{j+1 / 2}, \rho_{j}^{n}\right) v\left(c_{j+1 / 2}^{n}\right)\right)\right|+\frac{\tau}{6 \lambda} \sum_{j \in \mathbb{Z}}\left|D^{2} \rho_{j}^{n}\right| \\
\leq & \mathcal{C}(t) \tau
\end{aligned}
$$

where

$$
\begin{aligned}
\mathcal{C}(t)= & C\|v\|_{\mathbf{L}^{\infty}}\left\|\rho^{o}\right\|_{\mathbf{L}^{1}}+2\left\|\partial_{\rho} f\right\|_{\mathbf{L}^{\infty}}\left\|\rho^{o}\right\|_{\mathbf{L}^{1}}^{2}\left\|\eta^{\prime}\right\|_{\mathbf{L}^{\infty}}\left\|v^{\prime}\right\|_{\mathbf{L}^{\infty}} \\
& +\left(\left\|\partial_{\rho} f\right\|_{\mathbf{L}^{\infty}}\|v\|_{\mathbf{L}^{\infty}}+\frac{1}{3 \lambda}\right)\left(\mathcal{K}_{2} t+\sum_{j \in \mathbb{Z}}\left|\rho_{j+1}^{o}-\rho_{j}^{o}\right|\right) \mathrm{e}^{\mathcal{K}_{1} t}
\end{aligned}
$$

completing the proof.

Proof of Proposition 2.8. Fix $n \in \mathbb{N}$ and for any sequence $(\rho)_{j \in \mathbb{Z}}$ define the transformation $\rho \mapsto H(\rho)$ given by

$$
H_{j}^{n}(\rho)=\rho_{j}-\lambda\left(\mathbf{f}_{j+1 / 2}^{n}\left(\rho_{j}, \rho_{j+1}\right)-\mathbf{f}_{j-1 / 2}^{n}\left(\rho_{j-1}, \rho_{j}\right)\right),
$$

where the functions $\mathbf{f}_{j+1 / 2}^{n}$ are given by (2.5), but where, instead of $(2.6)$, the sequence $\left(c_{j+1 / 2}^{n}\right)_{j \in \mathbb{Z}}$ is now an arbitrary fixed sequence. Thus, $H_{j}^{n}(\rho)$ depends only on $\rho_{j-1}, \rho_{j}$ and $\rho_{j+1}$. Then, $H^{n}$ is monotone, in the sense that

$$
\frac{\partial H_{j}^{n}}{\partial \rho_{i}} \geq 0, \quad i=j-1, j, j+1
$$

The cases $i=j \pm 1$ are easily verified. If $i=j$, using (2.5) we find

$$
\begin{aligned}
\frac{\partial H_{j}^{n}}{\partial \rho_{j}} & =\frac{1}{3}-\frac{\lambda}{2}\left(\partial_{\rho} f\left(t^{n}, x_{j+1 / 2}, \rho_{j}\right) v\left(c_{j+1 / 2}\right)-\partial_{\rho} f\left(t^{n}, x_{j-1 / 2}, \rho_{j}\right) v\left(c_{j-1 / 2}\right)\right) \\
& \geq \frac{1}{3}-\lambda\left\|\partial_{\rho} f\right\|_{\mathbf{L}^{\infty}}\|v\|_{\mathbf{L}^{\infty}} \\
& \geq 0
\end{aligned}
$$

by the CFL condition (2.3). The definition (4.16) of $H^{n}$ and (2.8) imply that for any $k \in \mathbb{R}$

$$
\left|\rho_{j}-k\right|-\lambda\left(F_{j+1 / 2}^{k}\left(\rho_{j}, \rho_{j+1}\right)-F_{j-1 / 2}^{k}\left(\rho_{j-1}, \rho_{j}\right)\right)=H_{j}^{n}(\rho \wedge k)-H_{j}^{n}(\rho \vee k),
$$


where $k$ in the right-hand side above is understood as the sequence identically equal to $k$. The monotonicity condition (4.17) and the scheme (2.4)-(2.5) ensure that

$$
\begin{aligned}
& H_{j}^{n}(\rho \wedge k)-H_{j}^{n}(\rho \vee k) \\
\geq & H_{j}^{n}(\rho) \wedge H_{j}^{n}(k)-H_{j}^{n}(\rho) \vee H_{j}^{n}(k) \\
= & \operatorname{sgn}\left[H_{j}^{n}(\rho)-k+\lambda\left(f\left(t^{n}, x_{j+1 / 2}, k\right) v\left(c_{j+1 / 2}\right)-f\left(t^{n}, x_{j-1 / 2}, k\right) v\left(c_{j-1 / 2}\right)\right)\right] \\
& \times\left[H_{j}^{n}(\rho)-k+\lambda\left(f\left(t^{n}, x_{j+1 / 2}, k\right) v\left(c_{j+1 / 2}\right)-f\left(t^{n}, x_{j-1 / 2}, k\right) v\left(c_{j-1 / 2}\right)\right)\right] \\
\geq & \operatorname{sgn}\left(H_{j}^{n}(\rho)-k\right)\left[H_{j}^{n}(\rho)-k+\lambda\left(f\left(t^{n}, x_{j+1 / 2}, k\right) v\left(c_{j+1 / 2}\right)-f\left(t^{n}, x_{j-1 / 2}, k\right) v\left(c_{j-1 / 2}\right)\right)\right] \\
= & \left|H_{j}^{n}(\rho)-k\right|+\lambda \operatorname{sgn}\left[H_{j}^{n}(\rho)-k\right]\left[f\left(t^{n}, x_{j+1 / 2}, k\right) v\left(c_{j+1 / 2}\right)-f\left(t^{n}, x_{j-1 / 2}, k\right) v\left(c_{j+1 / 2}\right)\right] .
\end{aligned}
$$

In the last inequality we used also the non-negativity of the function $(a, b) \mapsto(\operatorname{sgn}(a+b)-\operatorname{sgn}(a))(a+b)$. From (4.18) and (4.19) we conclude that

$$
\begin{aligned}
\mid H_{j}^{n}(\rho)- & k|-| \rho_{j}-k \mid+\lambda\left(F_{j+1 / 2}^{k}\left(\rho_{j}, \rho_{j+1}\right)-F_{j-1 / 2}^{k}\left(\rho_{j-1}, \rho_{j}\right)\right) \\
& +\lambda \operatorname{sgn}\left(H_{j}^{n}(\rho)-k\right)\left(f\left(t^{n}, x_{j+1 / 2}, k\right)-f\left(t^{n}, x_{j-1 / 2}, k\right)\right) \leq 0 .
\end{aligned}
$$

Consider now the numerical approximation $\rho_{j}^{n}$ given by the algorithm (2.4). Then, we apply (4.20) to $\rho^{n}$, with the sequence $c_{j+1 / 2}$ appearing in (4.16) as given by the convolution (2.6). Observing that $H_{j}^{n}\left(\rho^{n}\right)=\rho_{j}^{n+1}$, we conclude that (2.9) holds.

Acknowledgements. The first author was partially supported by the Portuguese Foundation for Science and Technology (FCT) through the grant PTDC/MAT/110613/2009 - Nonlinear Hyperbolic Systems: Theory and Numerical Approximation, by PEst OE/MAT/UI0209/2011 and by the FCT through a Ciência 2008 fellowship. Part of this work was done while the first author was a researcher at CMAF, University of Lisbon.

\section{REFERENCES}

[1] S. Berres, R. Bürger, K.H. Karlsen and E.M. Tory, Strongly degenerate parabolic-hyperbolic systems modeling polydisperse sedimentation with compression. SIAM J. Appl. Math. 64 (2003) 41-80.

[2] F. Betancourt, R. Bürger, K.H. Karlsen and E.M. Tory, On nonlocal conservation laws modelling sedimentation. Nonlinearity 24 (2011) 855-885.

[3] A. Bressan, Hyperbolic systems of conservation laws. The one-dimensional Cauchy problem, vol. 20 of Oxford Lect. Ser. Math. Applications. Oxford University Press, Oxford (2000).

[4] F. Caetano, The linearization of a boundary value problem for a scalar conservation law. Commun. Math. Sci. 6 (2008) 651-667.

[5] J.A. Carrillo, R.M. Colombo, P. Gwiazda and A. Ulikowska, Structured populations, cell growth and measure valued balance laws. J. Differ. Eqs. 252 (2012) 3245-3277.

[6] R.M. Colombo, M. Garavello and M. Lécureux-Mercier, A class of nonlocal models for pedestrian traffic. Math. Models Methods Appl. Sci. 22 (2012) 1150023, 34.

[7] R.M. Colombo, M. Herty and M. Mercier, Control of the continuity equation with a non local flow. ESAIM: COCV 17 (2011) 353-379.

[8] R.M. Colombo and M. Lécureux-Mercier, Nonlocal Crowd Dynamics Models for Several Populations. Acta Math. Sci. Ser. B Engl. Ed. 32 (2012) 177-196.

[9] R.M. Colombo, M. Mercier and M.D. Rosini, Stability and total variation estimates on general scalar balance laws. Commun. Math. Sci. 7 (2009) 37-65.

[10] G. Crippa and M. Lécureux-Mercier, Existence and uniqueness of measure solutions for a system of continuity equations with non-local flow. Nonlinear Differ. Eqs. Appl. NoDEA (2012) 1-15.

[11] C.M. Dafermos, Hyperbolic conservation laws in continuum physics, vol. 325 of Grundlehren der Mathematischen Wissenschaften [Fundamental Principles of Mathematical Sciences]. Springer-Verlag, Berlin, 3rd edition (2010).

[12] R. Eftimie, Hyperbolic and kinetic models for self-organized biological aggregations and movement: a brief review. J. Math. Biol. 65 (2012) 35-75.

[13] A. Friedman, Conservation laws in mathematical biology. Discrete Contin. Dyn. Syst. 32 (2012) 3081-3097. 
[14] L. Gosse and F. James. Numerical approximations of one-dimensional linear conservation equations with discontinuous coefficients. Math. Comput. 69 987-1015, 2000.

[15] P. Gwiazda, T. Lorenz and A. Marciniak-Czochra, A nonlinear structured population model: Lipschitz continuity of measurevalued solutions with respect to model ingredients. J. Differ. Eqs. 248 (2010) 2703-2735.

[16] S. Gttlich, S. Hoher, P. Schindler, V. Schleper and A. Verl, Modeling, simulation and validation of material flow on conveyor belts. Prepint Reihe des Stuttgart Research Center for Simulation Technology, 746 (2013).

[17] K.H. Karlsen and N.H. Risebro, Convergence of finite difference schemes for viscous and inviscid conservation laws with rough coefficients. ESAIM: M2AN 35 (2001) 239-269.

[18] S.N. Kružhkov, First order quasilinear equations with several independent variables. Mat. Sb. (N.S.) 81 (1970) (123) $228-255$.

[19] R.J. LeVeque, Finite volume methods for hyperbolic problems. Cambridge Texts in Applied Mathematics. Cambridge University Press, Cambridge (2002).

[20] M.J. Lighthill and G.B. Whitham, On kinematic waves. II. A theory of traffic flow on long crowded roads. Proc. Roy. Soc. London Ser. A. 229 (1955) 317-345.

[21] J. Nieto, F. Poupaud and J. Soler, High-field limit for the Vlasov-Poisson-Fokker-Planck system. Arch. Ration. Mech. Anal. 158 (2001) 29-59.

[22] B. Piccoli and A. Tosin, Time-evolving measures and macroscopic modeling of pedestrian flow. Arch. Ration. Mech. Anal. 199 (2011) 707-738.

[23] P.I. Richards, Shock waves on the highway. Oper. Res. 4 (1956) 42-51. 